\title{
A linear-scaling method for non-covalent interactions
}

Robert Shaw, Grant Hill

Submitted date: 22/05/2019 Posted date: 22/05/2019

Licence: CC BY-NC-ND 4.0

Citation information: Shaw, Robert; Hill, Grant (2019): A linear-scaling method for non-covalent interactions. ChemRxiv. Preprint.

A novel method for the accurate and efficient calculation of interaction energies in weakly-bound complexes comprised of a large number of molecules is presented. The new ALMO+RPAd method circumvents the prohibitive scaling of coupled cluster singles and doubles while still providing similar accuracy across a diverse range of intermolecular interactions. Tests on various dimers and the S66 benchmark set demonstrate results within $0.5 \mathrm{kcal} / \mathrm{mol}$ of coupled cluster singles and doubles results. On a large cluster of water molecules, we achieve calculations involving over 3500 orbital and 12000 auxiliary basis functions in under ten minutes on a single CPU core.

File list (3)

almorpa.pdf (1.51 MiB)

view on ChemRxiv - download file

si.pdf $(337.70 \mathrm{KiB})$

view on ChemRxiv - download file 


\title{
A linear-scaling method for non-covalent
} interactions: An efficient combination of

\section{absolutely localized molecular orbitals and a local random phase approximation approach}

\author{
Robert A. Shaw and J. Grant Hill* \\ Department of Chemistry, University of Sheffield, Sheffield S3 7HF, U.K. \\ E-mail: grant.hill@sheffield.ac.uk
}

\begin{abstract}
A novel method for the accurate and efficient calculation of interaction energies in weakly-bound complexes comprised of a large number of molecules is presented. The new ALMO+RPAd method circumvents the prohibitive scaling of coupled cluster singles and doubles while still providing similar accuracy across a diverse range of intermolecular interactions. Linear-scaling procedures for the Fock build are given utilising absolutely localised molecular orbitals (ALMOs), resulting in the a priori exclusion of basis set superposition error. A bespoke data structure and algorithm using density fitting are described, leading to linear scaling for the storage and computation of the two-electron integrals. Electron correlation is included through a new, linear-scaling pairwise local random phase approximation approach, including exchange interactions, and decomposed into purely dispersive excitations (RPAxd). Collectively, these allow meaningful decomposition of the interaction energy into physically distinct contributions: electrostatic, polarisation, charge transfer, and dispersion. Comparison with
\end{abstract}


symmetry-adapted perturbation theory shows good qualitative agreement. Tests on various dimers and the S66 benchmark set demonstrate results within $0.5 \mathrm{kcal} \mathrm{mol}^{-1}$ of coupled cluster singles and doubles results. On a large cluster of water molecules, we achieve calculations involving over 3500 orbital and 12000 auxiliary basis functions in under ten minutes on a single CPU core.

\section{Introduction}

Chemistry relies on the idea that results obtained for molecules in one context are transferrable to other, similar situations. The idea of a molecule implies there is an inherent locality to the underlying description. While no such physical distinction truly exists, it is undeniably helpful to compartmentalize and categorize chemical systems. The latter is especially true in the case of non-covalent interactions, the ubiquitous forces underlying phenomena as diverse as geckos attaching themselves to walls, ${ }^{1,2}$ to the cohesion of asteroids, ${ }^{3}$ and more. ${ }^{4,5}$ Such interactions are defined to be any stabilization weaker than would be expected for a chemical bond - a definition so broad as to be insurmountably difficult to study without distinguishing further. Typically, such a distinction is found through decomposition of the interaction, into terms such as electrostatic, polarisation, and dispersion; ${ }^{6}$ the complex is then typified by the predominant component.

Non-covalent interactions are difficult to study, both experimentally and theoretically. Isolating weak forces in the condensed phase is problematic, ${ }^{7}$ although progress has been made in the use of molecular balances. ${ }^{8}$ Similarly, attempts in the gas phase are hindered by the fact that the thermal energy may overcome the weak binding. Theory therefore is of vital importance to understand these weakly-bound systems. However, the presence of small energy differences and the importance of dispersion - an effect entirely due to dynamical electron correlation - mean that only the most accurate computational methods perform consistently well. ${ }^{9-11}$ The lack of a reasonable description of dispersion in particular excludes the majority of density functionals, which have been shown to often give qualitatively and 
quantitatively inaccurate results, although progress has been made on this front. ${ }^{12-16}$ Even the cheaper correlated methods, especially second-order perturbation theory, will consistently overbind systems with significant delocalisation such as the benzene dimer, ${ }^{9}$ while underbinding many others, for example in saturated systems. ${ }^{17}$ The gold standard is considered to be coupled cluster with singles, doubles, and perturbative triples [CCSD $(\mathrm{T})],{ }^{18,19}$ which scales as $\mathcal{O}\left(N^{7}\right)$, where $N$ is a measure of system size. Problematically, the correlation energy generally converges very slowly with basis set size, such that very large bases are needed to achieve high accuracy. ${ }^{20}$ The result is that quantitative study is restricted to small molecular systems in the gas phase.

Considerable attention has been given to trying to reduce the cost of coupled cluster calculations. ${ }^{21-24}$ This has included the development of explicitly correlated F12 methods, which greatly accelerate convergence with respect to the size of the basis set. ${ }^{25-27}$ Density fitting of electron repulsion integrals (also referred to as resolution of the identity) has also been an key advance, ${ }^{28,29}$ making practical F12 calculations feasible and yielding considerable savings in computational effort in, for example, the Fock build ${ }^{30-32}$ and integral transformations. ${ }^{33-35}$ More recently, there have been attempts to use low-rank decompositions on the coupled cluster amplitude equations directly, with some promising early results. ${ }^{36}$ However, the prohibitive scaling behaviour of correlated wavefunction methods is unphysical. ${ }^{37}$ It is an artefact of the use of canonical molecular orbitals - the requirement for diagonalization results in orbitals extended over the entire system. As noted above, however, the properties of molecules are usually localised, and we would expect this to be reflected in the orbitals, at least in the occupied subspace. It is possible to use the invariance of the Hartree-Fock reference to rotations within the occupied and virtual subspaces to localise the molecular orbitals after the fact. ${ }^{38}$ When used subsequently in the correlated part of the calculation, this has two advantages: the pair correlation between distant, localized orbitals is small and so can either be treated more cheaply or not at all; the steep and unphysical expansion of the virtual space available to each electron can be eliminated by restricting excitations to atomic 
orbitals spatially close to the occupied molecular orbitals. There are now myriad such 'local correlation' methods, ${ }^{23,39-44}$ which allow for coupled cluster calculations that scale essentially linearly with system size. None of the orbital localization procedures used in local correlation approaches is perfect, however, and great care needs to be taken in choosing thresholds for when terms are neglected; in particular, a lack of consistency across different geometries could result in discontinuous potential energy surfaces.

The problems in performing high-accuracy calculations are further compounded by the fact that in practically interesting systems, e.g. biological and supramolecular contexts, complexes are not normally found in their equilibrium geometries. The interaction of interest will usually be strongly influenced by the environment, such that the surroundings must be taken into account. In large systems with multiple components, new difficulties present themselves: many-body effects become important, meaning that pair potential approximations are inadequate; ${ }^{45}$ the number of local minima increases exponentially with system size; ${ }^{46}$ the interand intra-molecular degrees of freedom become strongly coupled; ${ }^{47}$ and the gradients become tainted with basis set superposition errors (BSSE). ${ }^{48}$ The usual counterpoise scheme ${ }^{49}$ for correcting BSSE would involve calculations on all fragments in the full complex basis, in addition to the supramolecular calculation itself. The cost therefore explodes as the number of fragments increases. In addition, BSSE is larger for smaller basis sets, to the point where for very small bases it can be substantial enough as to result in a false minimum on a repulsive potential energy curve. ${ }^{50}$ Therefore, to study intermolecular interactions in large systems, it is preferable to eliminate the superposition errors explicitly from the beginning.

Several different methods have been suggested for the a priori elimination of BSSE. These include symmetry-adapted perturbation theory (SAPT), ${ }^{51-54}$ block-localized wavefunctions, ${ }^{55}$ absolutely/extremely localized molecular orbitals, ${ }^{56-58}$ local correlation methods, ${ }^{37,44,47,59,60}$ dual basis set methods, ${ }^{61-63}$ and the chemical Hamiltonian approach. ${ }^{50,64,65}$ The first of these is perhaps the most popular, and has the added advantage of providing a decomposition of the interaction energy into physical components, such as electrostatics 
and dispersion. However, the cost of high-order SAPT is not much better than traditional correlated methods, although recent density-fitted implementations are very efficient. 7,66 The chemical Hamiltonian approach, in contrast, attempts to directly identify the terms in the supermolecular Hamiltonian that introduce BSSE, and removes them. ${ }^{50,64,67}$ This has the advantage of being readily extendable to traditional correlated methods, in a way that SAPT cannot. The mean-field results obtained in this way have been seen to be virtually indistinguishable from counterpoise-corrected Hartree-Fock results, ${ }^{64}$ without the need for monomer calculations in the full basis. However, it offers no computational savings beyond this, and does not solve the underlying problem of localization.

An alternative method is to constrain the molecular orbitals (MOs) on a fragment to only use the atomic orbital (AO) basis local to that fragment. As the AOs are by construction localized, this results in absolutely localized molecular orbitals (ALMOs), with all BSSE necessarily eliminated as a result. This was first suggested by Stoll, ${ }^{68}$ then further developed by Cullen. ${ }^{69}$ It has sometimes been attributed to the later work of Gianinetti et al. under the name SCF for molecular interactions (SCF-MI); ${ }^{70,71}$ this was then extended by Iwata and coworkers. ${ }^{72-76}$ More recently, the Head-Gordon group have made extensive use of ALMOs, ${ }^{58,77}$ introducing charge transfer corrections and an energy decomposition analysis. ${ }^{78-81}$ Applications of the latter to a wide range of systems have yielded interesting results. ${ }^{82,83}$

In the present work, we will use absolutely localized molecular orbitals to develop an inherently fragment-based method for calculating interaction energies free from BSSE. These will be used to eliminate the localization problems in a local treatment of the correlation energy, and in this way naturally get a correlated energy decomposition analysis. Following development of the theory, we will demonstrate how the new method can be made to be essentially linear scaling in the number of fragments, and described its amenability to massive parallelization. Finally, the applicability and efficiency of the method will be demonstrated on a range of chemically interesting systems, showing that it gives essentially coupled cluster quality results at a fraction of the computational cost. 


\section{Theory}

The theory behind absolutely localized molecular orbitals has been developed, and redeveloped, by several groups. We present it here briefly in the context of second quantization, as this will be helpful when including electron correlation later in the theory.

Consider a system of $F$ fragments. Each fragment, $X$, has an atomic orbital (AO) basis, $\left\{\chi_{X \mu}\right\}$, associated with it, containing $n_{X}$ functions. From these, $o_{X}$ occupied and $v_{X}$ virtual molecular orbitals, $\phi_{X p}$, will be formed. The total supermolecular basis then comprises the union of all such fragment bases, with $N=\sum_{X} n_{X}$ AOs, $O=\sum_{X} o_{X}$ occupied orbitals, and $V=\sum_{X} v_{X}$ virtual orbitals. Throughout the following, we define

$$
n / o / v=\max _{X=1,2, \ldots, F}\left\{n_{X} / o_{X} / v_{X}\right\}
$$

Tensor notation is used throughout, as is the Einstein summation convention with the exception of sums over fragments, which will always be explicitly shown for clarity. As is usual, lowered (subscripted) indices indicate covariant quantities, while raised (superscripted) indices represent contravariant quantities. Dots are used as placeholder indices given the need to use composite indices to denote the fragmentation; for example, $X i$ is a single index.

\subsection{Absolutely localized molecular orbitals (ALMOs)}

Following the usual self-consistent field (SCF) approach, the covariant MOs are expanded in the basis of AOs, but in this case with these being fragment-localised:

$$
\left|\phi_{X i}\right\rangle=\left|\phi_{X \mu}\right\rangle T_{\cdot X i}^{X \mu \cdot} \text { and }\left|\phi_{X a}\right\rangle=\left|\phi_{X \mu}\right\rangle V_{\cdot X a}^{X \mu}
$$

where the coefficient tensors, $T$ and $V$, are constrained such that

$$
T_{\cdot Y i}^{X \mu \cdot}=V_{\cdot X i}^{Y \mu \cdot}=0 \text { if } X \neq Y
$$


This is in contrast to the canonical theory, where we would instead constrain all MOs to be orthogonal; the above removes the degrees of freedom that would allow us to do so, such that the orbitals so obtained are in general nonorthogonal.

The wavefunction is more compactly represented in second quantization by operators, $a_{p \gamma}^{\dagger}$ and $a_{p \gamma}$, which create or annihilate, respectively, an electron in orbital $\phi_{p}$ with spin $\gamma$. The lack of orthogonality means that these satisfy the anticommutation relations

$$
\begin{aligned}
& {\left[a_{p \gamma_{1}}^{\dagger}, a_{q \gamma_{2}}^{\dagger}\right]_{+}=\left[a_{p \gamma_{1}}, a_{q \gamma_{2}}\right]_{+}=0} \\
& {\left[a_{p \gamma_{1}}^{\dagger}, a_{q \gamma_{2}}\right]_{+}=\delta_{\gamma_{1} \gamma_{2}} s_{p q}}
\end{aligned}
$$

where $s_{p q}=\left\langle\phi_{p} \mid \phi_{q}\right\rangle$. This completely encodes the antisymmetry of the wavefunction, but the non-vanishing nature of $s_{p q}$ complicates matters. Instead, we introduce a symmetrically orthogonalised set of operators for the occupied orbitals as:

$$
\tilde{a}_{i \gamma}^{\dagger}=a_{j \gamma}^{\dagger}\left[\sigma^{-1 / 2}\right]^{j i}
$$

where $\sigma$ is the occupied-occupied overlap metric. The density operator, which projects onto the occupied subspace, is thus $\hat{\rho}=\left|\tilde{\phi}_{i}\right\rangle\left\langle\tilde{\phi}_{i}\right|$; we transform the virtual orbitals by projecting them out of the occupied subspace:

$$
\tilde{a}_{a \gamma}^{\dagger}=(\hat{1}-\hat{\rho}) a_{a \gamma}^{\dagger}=\hat{q} a_{a \gamma}^{\dagger}
$$

The occupied-occupied and occupied-virtual anticommutation relations thus become the usual:

$$
\left[\tilde{a}_{i \gamma_{1}}^{\dagger}, \tilde{a}_{q \gamma_{2}}\right]_{+}=\delta_{\gamma_{1} \gamma_{2}} \delta_{i q}
$$

Writing a single excitation operator in the form $\tilde{E}_{p q}=\sum_{\gamma} \tilde{a}_{p \gamma}^{\dagger} \tilde{a}_{q \gamma}$ the usual molecular 
Hamiltonian is given by:

$$
\hat{H}=\tilde{h}_{p q} \tilde{E}_{p q}+\frac{1}{2} g_{p q r s}\left(\tilde{E}_{p q} \tilde{E}_{r s}-\delta_{p s} \tilde{E}_{r q}\right)
$$

where $\tilde{h}_{p q}$ and $\tilde{g}_{p q r s}$ are the one- and two-electron integrals in the orthogonalized basis. For simplicity, we will consider only a restricted closed-shell wavefunction, $|\Phi\rangle=\prod_{i \in \text { occ. }} a_{i \alpha}^{\dagger} a_{i \beta}^{\dagger}|\rangle$, but the extension to the spin-unrestricted case is simple, as has been detailed elsewhere. ${ }^{84}$ The energy is thus given by

$$
E=\langle\tilde{i}|\hat{h}+\hat{f}| \tilde{i}\rangle=\operatorname{Tr}\{(\mathbf{H}+\mathbf{F}) \mathbf{P}\}
$$

where $\mathbf{H}$ and $\mathbf{F}$ are the $\mathrm{AO}$ representations of the core Hamiltonian and Fock operators, $\hat{h}$ and $\hat{f}$, with $F_{\mu \nu}=H_{\mu \nu}+\langle\mu \nu \| \lambda \tau\rangle P_{\tau \lambda}$. From this we see that the density matrix is given by

$$
P^{X \mu, Y \nu}=T_{\cdot X i}^{X \mu \cdot \sigma^{X i, Y j}} T_{Y j}^{Y \nu}
$$

Note that this couples fragments together, such that the energy is not simply a sum of disjoint monomer energies.

Taking the derivative of the energy with respect to a rotation, $e^{-\hat{\kappa}}$, of the orthogonalized orbitals, where $\hat{\kappa}=\kappa_{p q}\left(\tilde{E}_{p q}-\tilde{E}_{q p}\right)$, we get the stationary condition

$$
\frac{\partial E}{\partial \kappa_{a i}}=2\left\langle\Phi\left|\left[\tilde{E}_{a i}, \hat{H}\right]\right| \Phi\right\rangle=4\left\langle\tilde{\phi}_{a}|\hat{f}| \tilde{\phi}_{i}\right\rangle=4\left\langle\phi_{X a}|\hat{q} \hat{f}| \tilde{\phi}^{X i}\right\rangle=0
$$

This is equivalent to the requirement $\hat{q} \hat{f} \hat{\rho}\left|\tilde{\phi}^{X i}\right\rangle=0$. Thus occupied-occupied and virtualvirtual rotations within each fragment's orbital space do not affect the energy, such that we may choose orbitals within each fragment to be orthonormal, diagonalising the on-fragment blocks of the Fock matrix: $\left\langle\tilde{\phi}_{X j}|\hat{f}| \tilde{\phi}^{X i}\right\rangle=\epsilon_{X i} \delta_{i j}$. We therefore define fragment-localised 
density and Fock operators as

$$
\hat{\rho}_{X}=\left|\tilde{\phi}^{X i}\right\rangle\left\langle\tilde{\phi}_{X i}\right| \text { and } \hat{f}_{X}=\left(\hat{q}+\hat{\rho}_{X}^{\dagger}\right) \hat{f}\left(\hat{q}+\hat{\rho}_{X}\right)
$$

Note that $\hat{\rho}=\sum_{X} \hat{\rho}_{X}$, and that $\hat{f}_{X}$ has been constructed to be Hermitian. Using the condition in Equation 9, along with the facts that $\hat{\rho}_{X}\left|\phi_{Y i}\right\rangle=\delta_{X Y}\left|\tilde{\phi}^{X i}\right\rangle$ and $\hat{q}\left|\phi_{X i}\right\rangle=0$, we see that

$$
\hat{f}_{X}\left|\phi_{X i}\right\rangle=\left|\phi_{X j}\right\rangle\left\langle\tilde{\phi}_{X j}|\hat{f}| \tilde{\phi}^{X i}\right\rangle=\left|\phi_{X i}\right\rangle \epsilon_{X i}
$$

Transferring to the AO representation, this yields a set of modified Roothaan-Hall equations for each fragment block:

$$
\mathbf{F}_{X} \mathbf{C}_{X}^{X}=\mathbf{S}_{X X} \mathbf{C}_{X}^{X} \epsilon_{X}
$$

By construction, there can be no BSSE present in the solution to these. Moreover, given that the diagonalization of an $M \times M$ matrix is an $\mathcal{O}\left(M^{3}\right)$ process, the cost for diagonalising $F$ fragment Fock matrices is $\mathcal{O}\left(F n^{3}\right)$, as opposed to $\mathcal{O}\left(N^{3}\right)=\mathcal{O}\left(F^{3} n^{3}\right)$ for the full matrix.

\subsection{Perturbative correction to the ALMO SCF energy}

By restricting the orbitals to be absolutely localized, the energy obtained must in general be higher than the full HF solution. The missing density can be attributed to the lack of orbital rotations between fragments, i.e. the lack of density transfer between fragments. In fact, a Mulliken analysis ${ }^{85}$ trivially shows that the charge on each fragment remains static under the ALMO approximation; we note, however, that this is not true using a Löwdin analysis, ${ }^{86}$ implying there is still a degree of uncertainty.

Equivalently, the difference can be thought of as the monomers each being restricted to a smaller, local AO basis instead of being allowed to relax in the full supermolecular basis. Dual-basis methods offer an efficient way to expand the converged solution into the larger basis. ${ }^{87-90}$ In this approach, we diagonalize the Fock matrix and construct a new density, 
$\mathbf{P}_{\infty}$, from which the energy correction is determined as

$$
\Delta E=\operatorname{Tr}\left\{\mathbf{F}(\mathbf{P})\left[\mathbf{P}_{\infty}-\mathbf{P}\right]\right\}
$$

However, diagonalization of the full Fock matrix is a costly $\mathcal{O}\left(N^{3}\right)$ procedure. Instead, it can be circumvented by iteratively finding an orbital rotation, $\hat{u}$, that diagonalises the Fock operator, as outlined by Liang and Head-Gordon. ${ }^{91}$ Defining $\mathbf{x}_{v o}=\mathbf{u}_{v o} \mathbf{u}_{o o}^{-1}$, where the $o$ and $v$ subscripts denote occupied and virtual blocks, the energy correction is simply given by:

$$
\Delta E=2 \operatorname{Tr}\left\{\mathbf{x}_{v o} \mathbf{f}_{o v}\right\}
$$

The rotation parameters are found by iterative solution of

$$
\mathbf{f}_{v o}+\mathbf{f}_{v v} \mathbf{x}_{v o}-\mathbf{x}_{v o} \mathbf{f}_{o o}-\mathbf{x}_{v o} \mathbf{f}_{v o} \mathbf{x}_{v o}=0
$$

This formulation is useful for two main reasons. Firstly, its most expensive part computationally is the quadratic term, involving two matrix-matrix multiplications. It therefore scales an order of magnitude better than diagonalization, and despite being iterative, involves far fewer floating point operations. It should be noted that the quadratic term can be dispensed with, leading to the usual second-order peturbative correction, removing the need for iterations. However, the second advantage of the present approach will be seen later in its utility for decomposing the energy correction into contributions between fragments.

\subsection{Including electron correlation}

As discussed in the introduction, dispersion is often very important in intermolecular interactions. ${ }^{7}$ Dispersion can be identified with dynamical electron correlation, of which only exchange terms are included in the mean-field treatment above. As such, it is vital to go beyond the mean-field limit if we are to treat supermolecular systems accurately. It is 
well-known that dispersion can be formulated exactly in terms of the frequency-dependent electric polarizabilities of a system, using a technique called the 'adiabatic connection'. ${ }^{92-94}$ In simple terms, the Hamiltonian in Equation 6 is decomposed into a zeroth-order part (the Fock operator) and a fluctuation potential, $\hat{w}$, controlled by a parameter $\lambda: \hat{H}=\hat{f}+\lambda \hat{w}$. By slowly, or adiabatically, 'switching on' the perturbation from $\lambda=0$ to $\lambda=1$ and integrating, the correlation is recovered. If we make the assumption that only the part of the response of the electrons to a light wave that is in phase with the wave makes a non-negligible contribution, we arrive at the random phase approximation (RPA). ${ }^{95-98}$ The correlation energy is then found by integrating the fluctuation over the response in the electric polarizabilities, themselves determined by solution of a Dyson equation. This is the approach taken both in time-dependent and range-separated density functional theory. ${ }^{92,94,99-104}$ In this way, it can be shown that the correct $R^{-6}$ long-range dispersive behaviour in the interaction energy is recovered. ${ }^{6}$

More recent interest in RPA has arisen from its intimate connection with coupled cluster methods. ${ }^{105-110}$ In fact, direct RPA (dRPA), ${ }^{111}$ where the fluctuation $w_{i a j b}$ is simply the two-electron integral $(i a \mid j b)$, is entirely equivalent to coupled cluster doubles (CCD) with only the diagrams with ring topologies included. ${ }^{108}$ This is termed ring coupled cluster, or rCCD. Starting from the orthogonalized basis of Equation 4, we further orthogonalize the virtual orbitals within themselves in the same way:

$$
\tilde{a}_{a \gamma}=(\hat{1}-\hat{\rho}) a_{b \gamma}\left[\pi^{-1 / 2}\right]^{b a}
$$

where $\pi$ is the virtual-virtual overlap metric. Note that the projection out of the occupied space necessarily delocalizes the virtual orbitals somewhat, but this can be shown to be in the sense that leaves them closest to the original, absolutely localized orbitals. ${ }^{112}$ They are therefore expected to remain well localized. The double excitation operator $\hat{T}_{2}$ can then be written as $\tilde{t}_{i j}^{a b} \tilde{a}_{a}^{\dagger} \tilde{a}_{b}^{\dagger} \tilde{a}_{j} \tilde{a}_{i}$ where $\tilde{t}_{i j}^{a b}$ are the excitation amplitudes. Writing $\left|\Phi_{i j}^{a b}\right\rangle=\tilde{a}_{a}^{\dagger} \tilde{a}_{b}^{\dagger} \tilde{a}_{j} \tilde{a}_{i}|\Phi\rangle$, 
the CCD energy and amplitude equations are given by:

$$
\begin{aligned}
\Delta E_{\mathrm{CCD}} & =\left\langle\Phi\left|\hat{H} \exp \left(\hat{T}_{2}\right)\right| \Phi\right\rangle=\tilde{w}_{i a j b} \tilde{t}_{i j}^{a b} \\
& \left\langle\Phi_{i j}^{a b}\left|\hat{H} \exp \left(\hat{T}_{2}\right)\right| \Phi\right\rangle=E_{\mathrm{CCD}}\left\langle\Phi_{i j}^{a b}\left|\exp \left(\hat{T}_{2}\right)\right| \Phi\right\rangle
\end{aligned}
$$

It can easily be shown ${ }^{108}$ diagrammatically that the above amplitude equation with only ring diagrams retained leads to the dRPA residual:

$$
\tilde{\mathbf{R}}=\tilde{\mathbf{K}}+\tilde{\mathbf{A}} \tilde{\mathbf{t}}+\tilde{\mathbf{t}} \tilde{\mathbf{A}}+\tilde{\mathbf{t}} \tilde{\mathbf{K}} \tilde{\mathbf{t}}=0
$$

Here, $\tilde{K}_{i a j b}=(\tilde{i} \tilde{a} \mid \tilde{j} \tilde{b})$, and $\mathbf{A}=\tilde{\epsilon}+\mathbf{K}$, where $\tilde{\epsilon}_{i a j b}=\delta_{i j} \tilde{f}_{a b}-\tilde{f}_{i j} \delta_{a b}$. This is an algebraic Riccatti equation, for which many simple and efficient methods of solution exist, ${ }^{113}$ allowing iterative determination of the RPA energy, given from Equation 15 as

$$
\Delta E_{\mathrm{dRPA}}=\frac{1}{2} \operatorname{Tr}\{\tilde{\mathbf{K}} \tilde{\mathbf{t}}\}
$$

However, we would like to make use of the inherent locality of the orbitals, achieving a local correlation method with the orbitals localized a priori. To do so, we back-transform Equations 16 and 17 using the relations in Equations 4 and 14, to get the following:

$$
\begin{aligned}
\Delta E_{\mathrm{dRPA}} & =\frac{1}{2}\left[\sigma^{i k} \pi^{a c} K_{k c l d} \pi^{d b} \sigma^{l j}\right] t_{i j}^{a b} \\
R_{i a j b} & =K_{i a j b}+A_{i a k c} \sigma^{k l} \pi^{c d} t_{l j}^{d b}+t_{i k}^{a c} \pi^{c d} \sigma^{k l} A_{l d j b}+t_{i k}^{a c} \pi^{c d} \sigma^{k l} K_{l d m e} \sigma^{m n} \pi^{e f} t_{n j}^{f b}=0
\end{aligned}
$$

This will allow us both to make considerable computational savings, and to decompose the dispersion energy into contributions between fragments.

\subsubsection{Including exchange}

Direct RPA performs very well in the long range, but provides a much poorer description of electron correlation in the short range. ${ }^{110,114,115}$ For example, the pair-correlation function 
in the high-density electron gas becomes negative at small separations. ${ }^{115,116}$ This is due to the missing exchange terms in the Coulomb interaction, i.e. the fact that antisymmetrized integrals are not used. At short separations, exchange-repulsion dominates as the distributions of electrons of the same spin attempt to overlap. Several different schemes have been suggested to ameliorate this problem, ${ }^{110,114,116}$ one of the simplest of which is to simply calculate the energy by contracting the amplitudes with the antisymmetrized integrals:

$$
B_{i a j b}=2(i a \mid j b)-(i b \mid j a)
$$

This is called the second-order screened exchange (SOSEX) correction. ${ }^{117}$ Such a correction only affects the energy, however, whereas a full solution requires inclusion of exchange effects in the residuals. By replacing all instances of $\mathbf{K}$ with $\mathbf{B}$ in Equation 16 (and the equivalent local forms), we arrive at the RPA plus exchange (RPAx) version. ${ }^{110}$ The energy becomes

$$
\Delta E_{\mathrm{RPAx}}=\frac{1}{4} \operatorname{Tr}\{\tilde{\mathbf{B}} \tilde{\mathbf{t}}\}
$$

where the extra factor of $1 / 2$ is necessary to avoid double counting. SOSEX is equivalent to including all contractions of the amplitudes with the interaction that does not form 'loops', i.e. where exactly one particle and one hole line have been exchanged, whereas RPAx also includes exchange in the Coulombic screening when determining the amplitudes. We note that the second-order contribution in RPAx equates to the MP2 energy.

\subsection{Energy decomposition analysis}

The motivation for the choice of development of the method outlined above has been twofold: to achieve linear scaling of computation cost with respect to the number of fragments, as will be discussed in the next section; and to yield a physically motivated energy decomposition. Building on previous work using ALMOs, ${ }^{78,79}$ we describe four main components.

The first of these is the 'frozen' energy, describing the electrostatic interaction between 
the unrelaxed, frozen monomer orbitals in their distorted geometries. After the full SCF calculations have been performed on the monomers in their interacting geometries, each fragment has a set of unperturbed occupied orbitals, $\mathbf{T}_{X, \text { frz. }}$. Inserting these in Equation 8 gives the frozen density, which due to the presence of the inverse metric is in general not the superposition of non-interacting fragment densities, as the orbitals will not be orthogonal between fragments. Using this density in Equation 7 gives the total energy of the unperturbed system; the difference between this and the sum of the monomer energies is then defined to be the frozen energy. This therefore represents the interactions between unperturbed fragment electron densities, represented by the occupied orbitals, which in turn corresponds to multipole-multipole interactions. A further term, the relaxation energy, could be included at this point as the difference between the monomer energies in their relaxed and interacting geometries.

The next two terms are polarization and charge transfer. During the ALMO SCF procedure, the frozen orbitals above are allowed to relax, corresponding to the distortion of the monomer densities due to the presence of other fragments, i.e. polarization. The polarization energy can thus be defined as the difference between the energy calculated with the frozen and full densities. However, as was noted earlier, the absolute localization constraint prevents transfer of electron density between fragments. Allowing the orbitals to relax in the full basis, as described by Equation 12, can therefore be robustly defined as representing charge transfer. Charge transfer is by definition a directional quantity, however, and it is desirable to be able to determine the contribution from 'charge' transferred between fragments $X$ and $Y$. We do this by introducing Mulliken partition operators:

$$
\hat{p}_{X i}=\left|\tilde{\phi}_{X i}\right\rangle\left\langle\tilde{\phi}^{X i}\right| \text { and } \hat{q}_{X a}=\left|\tilde{\phi}_{X a}\right\rangle\left\langle\tilde{\phi}^{X a}\right|
$$

noting that the sum over all fragments and orbitals of these gives the ALMO occupied (density) and virtual subspace projectors, respectively. The energy due to charge transfer 
between fragments $X$ and $Y$ is then found by inserting these into Equation 12:

$$
\Delta E_{\mathrm{CT}}^{X \rightarrow Y}=\sum_{i, a} \operatorname{Tr}\left\{\hat{p}_{X i} \hat{f} \hat{q}_{Y a} \hat{x}\right\}=\operatorname{Tr}\left\{\left(\mathbf{f}_{o v}\right)_{X Y}\left(\mathbf{x}_{v o}\right)_{Y X}\right\}
$$

This highlights the usefulness of taking the iterative approach of Equation 13. We note that, whereas the frozen and polarization energies are constructed to be free from BSSE, the charge transfer term necessarily reintroduces some error. However, due to the perturbative nature of the correction, this error is expected to be small, as will be shown later.

By transforming the density in the same way, we can also define (Mulliken) partial charges:

$$
\Delta Q_{\mathrm{CT}, \mathrm{M}}^{X \rightarrow Y}=\operatorname{Tr}\left\{\mathbf{T}_{X}^{\dagger} \mathbf{P} \tilde{\mathbf{V}}_{Y}\left(\mathbf{x}_{v o}\right)_{Y X}\right\}
$$

However, we stress that this does not represent a physical transfer of electrons from one entity to another, it is rather the non-local response of the electron density on each fragment when brought into proximity with the other fragments. That is, we have split polarization into local and non-local portions; $\Delta E_{\text {pol. }}$ reflects the redistribution of electron density constrained to a finite volume around the fragment, defined by the extent of the local atomic orbitals, while $\Delta E_{\mathrm{CT}}$ encompasses the remaining relaxation.

The dynamical electron correlation in this method is determined using RPA, which encompasses to some extent infinite-order many-body effects on the single particle level. The $a$ priori local nature of the orbitals allows for considerable savings in computational cost, but also for the explicit removal of BSSE from the correlation energy. Solving Equations 18 (or the RPAx equivalent) leads to excitation amplitudes in terms of orbitals strictly localized to specific fragments. As only double excitations are included in rCCD, such excitations can involve at most four fragments (one for each index). Extending an idea first introduced by Schütz et al. in the context of local MP2, ${ }^{47}$ we can classify these excitations as described in Figure 1.

We identify five categories of excitation, based on what they physically represent. When 


\section{Two fragments}

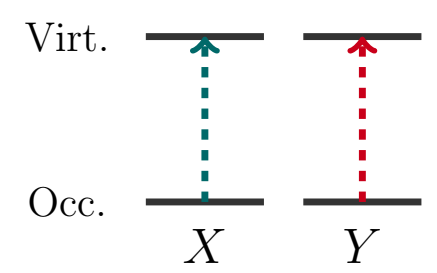

(a) Dispersion

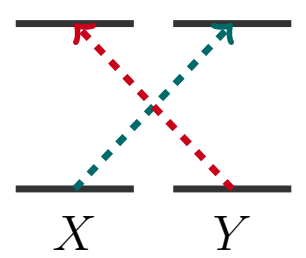

(b) Exch.-dispersion

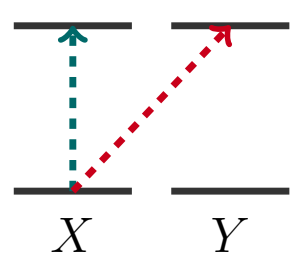

(c) Ionic

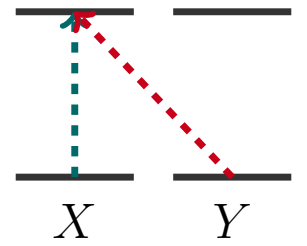

(d) Ionic

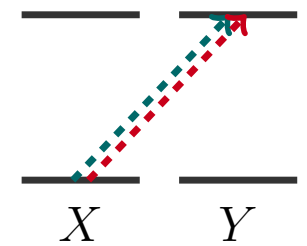

(e) BSSE

\section{Three fragments}

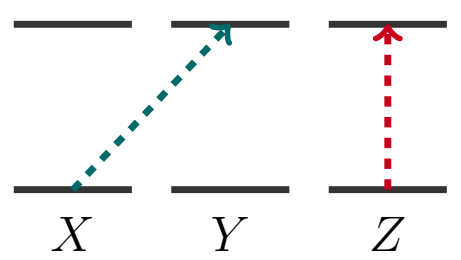

(f) Ionic

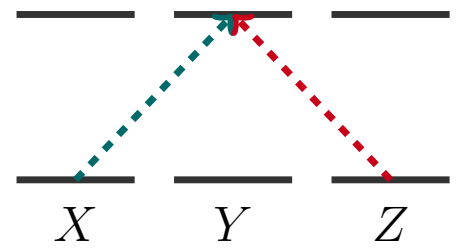

(h) Ionic

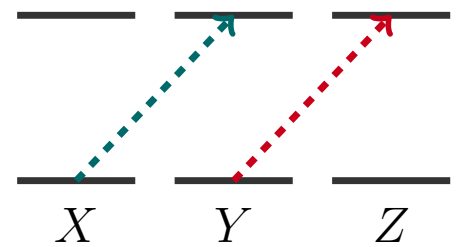

(g) Ionic

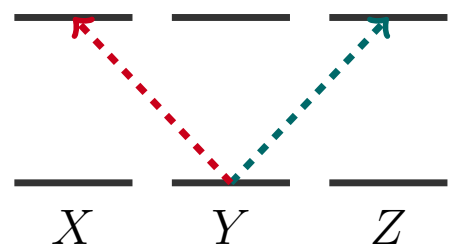

(i) BSSE

Four fragments

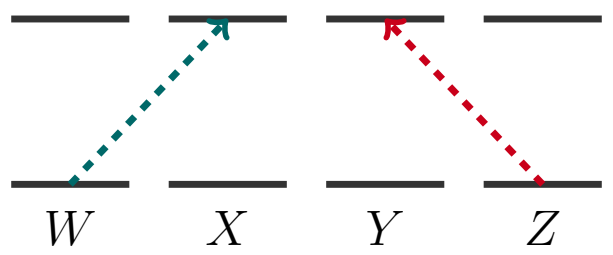

(j) Ionic

Figure 1: Schematics of all unique classes of inter-fragment excitations. 
all of the orbitals are located on a single fragment, not shown in the Figure, a term is clearly intramolecular. As these will only be slightly perturbed by the presence of the rest of the complex, it is reasonable to approximate that these do not form part of the interaction energy. Dispersion is taken to be the response of on-fragment excitations to simultaneous excitation within a separate fragment, i.e. the dispersive coupling between the separated fragment electron densities. This corresponds to class (a) in the Figure, whereas class (b) is exchangedispersion. This is again a number-conserving coupling of excitations involving exchange between fragments. This is not well-separated from the dispersion term, as the reference wavefunction is fully antisymmetrised and so inherently includes exchange-repulsion in all terms. As such, this is not directly comparable with the same term in SAPT. The dispersive energy contributions are therefore taken from Equation 18 as:

$$
\begin{aligned}
\Delta E_{\text {disp. }} & =\sum_{X} \sum_{Y>X} \sum_{(i a) \in X} \sum_{(j b) \in Y} t_{i j}^{a b}\left[\sigma^{-1} \pi^{-1} K \pi^{-1} \sigma^{-1}\right]_{i j}^{a b} \\
\Delta E_{\text {exch-disp. }} & =\sum_{X} \sum_{Y>X} \sum_{(i b) \in X} \sum_{(j a) \in Y} t_{i j}^{a b}\left[\sigma^{-1} \pi^{-1} K \pi^{-1} \sigma^{-1}\right]_{i j}^{a b}
\end{aligned}
$$

The two remaining classes in Figure 1 are ionic and BSSE, all of which involve excitations from $X$ to $Y$, without a corresponding excitation into $X$. It should be stressed that no electrons are transferred; these are rather couplings between different states in the underlying Fock space. The term ionic is denoting that the excitations are not particle number-conserving within a fragment, representing another form of 'charge transfer'. In particular, it is supposed that diagrams (e) and (i), which are double excitations from the same fragment into distant virtual orbitals on separate fragments, represent an attempt to improve the description of intramolecular correlation using the extended basis. In principle, any terms involving excitations between fragments could contain some measure of superposition error, but the aforementioned terms are likely to be the main contributors, due to the sharp distance dependence of the underlying physical interactions.

As the RPA is performed on the ALMO density directly - no charge-transfer correction is 
made to the wavefunction, only the energy - the ionic terms will likely contain not only some BSSE, but also a significant amount of the charge transfer effects that would be encompassed at the mean-field level by $\Delta E_{\mathrm{CT}}$. In addition, the virtual orbitals are projected out of the occupied subspace, as per Equations 5 and 14, such that they are not completely localised. As a result, the ionic and BSSE terms are entangled, albeit only to a small extent; coupled with the fact that superposition errors are known to be much larger at the correlated as opposed to mean-field level, we choose to define the charge-transfer contribution as in Equation 12, and neglect all but the dispersion and exchange-dispersion terms in Figure 1.

\section{Implementation}

The main aim of the present work is to present an accurate method for non-covalent interactions that is efficient and scalable to large systems. As we have described the system in terms of fragments, there are two scaling regimes to consider: scaling with fragment size, and with the number of fragments. The latter magnifies the former considerably, and therefore is the larger source of computation cost; effectively it is a measure of the entire system size. As such, we focus here on achieving linear scaling with regards to the number of fragments. There are three main expensive parts of any SCF procedure: evaluation (and storage) of the two-electron integrals; forming the Fock matrix; diagonalizing the Fock matrix. The latter exhibits the correct scaling by virtue of the use of ALMOs, as was mentioned earlier. In the RPA portion of the calculation, the main difficulties are in the transformation of the integrals to the molecular orbital basis, and the solution of the amplitude equations.

\subsection{Density fitting}

Both problems involving the two-electron integrals can be ameliorated by using density fitting $(\mathrm{DF})$. This requires the evaluation of two- and three-center Coulomb integrals, $G_{P Q}=(P \mid Q)$ and $B_{\mu \nu}^{P}=(\mu \nu \mid P)$, where $\left\{\chi_{P}\right\}$ is an auxiliary basis set (ABS) of Gaussians. We use capital 
letters to denote functions of the ABS, and lowercase as per usual for the MO basis. The usual algorithm ${ }^{30}$ for the evaluation and transformation of the density-fitted integrals is to first Cholesky decompose $\mathbf{G}=\mathbf{L L}^{\mathrm{T}}$ and invert the Cholesky factor, $\mathbf{L}$, before forming $\mathbf{b}=\mathbf{B L}^{-1}$. The transformed integrals, $\tilde{\mathbf{b}}$ can thus be formed in two steps as

$$
\tilde{b}_{i a}^{P}=\sum_{\nu}\left(\sum_{\mu} b_{\mu \nu}^{P} T_{\mu}^{i}\right) V_{\nu}^{a}
$$

If the full integrals are then required, they can be formed in the $\mathrm{AO}$ or MO bases as $\mathbf{b b}^{T}$ or $\tilde{\mathbf{b}} \tilde{\mathbf{b}}^{T}$, respectively.

The evaluation of $\mathbf{B}$ clearly requires $\mathcal{O}\left(N^{2} M\right)$ computations, where $N$ and $M$ are the number of functions in the orbital and auxiliary bases, respectively. The Cholesky decomposition is $\mathcal{O}\left(M^{3}\right)$ but with a very small prefactor, while the transformation is $\mathcal{O}\left(O N^{2}\right)$ for the inner step and $\mathcal{O}(O V N)$ for the outer step. Overall the algorithm is therefore cubic scaling, as is the memory cost. While this is a considerable improvement on the quartic and quintic scalings of the conventional integral and transformation algorithms, it is still problematic for large systems.

However, such steep scaling with system size is unphysical. If we consider two oneparticle densities separated by a distance $R$, represented by Gaussians of combined angular momentum $l$ and exponents $\alpha_{p}, \alpha_{q}$, it can be shown ${ }^{118}$ that the electron repulsion integral, $V$, between the two asymptotically follows the relation

$$
V_{p q} \sim \frac{2}{\alpha_{p q}^{n} R^{2 n+1}} \text { where } \alpha=\frac{\alpha_{p} \alpha_{q}}{\alpha_{p}+\alpha_{q}}
$$

Hence the worst case is for spherical Gaussians, where the integral decays at the same rate as the Coulombic potential itself. Nonetheless, the integral tensors should eventually become highly sparse, with each fragment having an effectively fixed domain size over which integrals do not fall below a given threshold.

One possibility for exploiting this would be to directly use conventional sparse matrix 


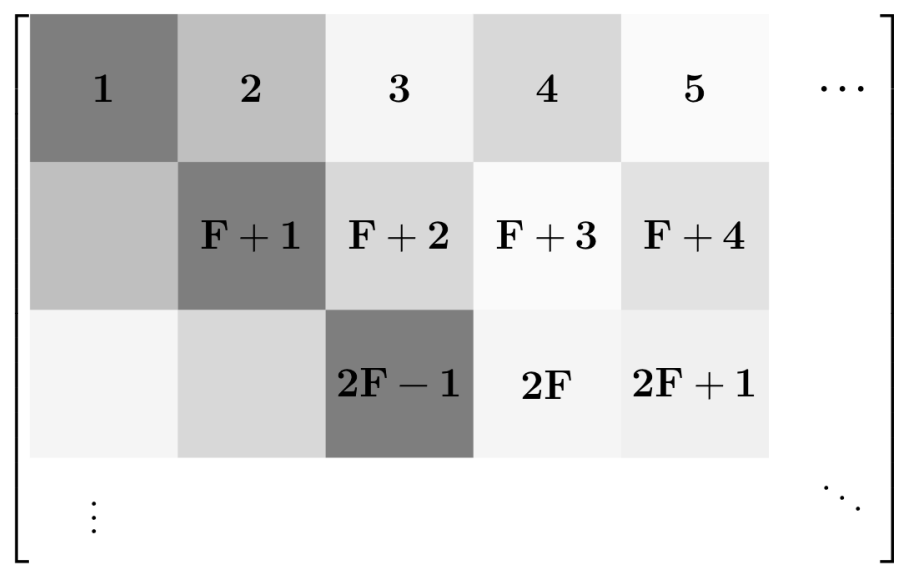

Figure 2: Schematic of a simple sparse matrix representation as fragment-based blocks, where $F$ is the number of fragments. The darkness of a block indicates the magnitude of the maximum integral element within that block, a measure of which blocks can be ignored. For example, all diagonal blocks will necessarily be 'dark' and need to be included, whereas most off-diagonal blocks (such as blocks 3 and 5 on the top row) are negligible.

formats, such as the popular compressed row storage data structure. ${ }^{119}$ However, this is not useful if element-wise access is needed, as this would require expensive binary searches. To solve this problem, we have devised an adapted sparse data structure and accompanying algorithm, which fully utilises the underlying fragmented structure of the system. Consider a slice of the three-index DF tensor $\mathbf{B}$ along the ABS axis, $P$. The matrix can be represented as shown in Figure 2, where a typical sparsity pattern is also indicated, and the blocks are fragment by fragment. This matrix can be represented by two arrays with length $N_{\text {Blocks }}=$ $F(F+1) / 2$, after symmetry is taken into account. The first is an array of zero-sized matrices, while the second is a list of boolean values reflecting whether the corresponding integral block is zero. The approach can trivially be expanded to include the ABS blocks by 'z-marching' along the index $P$ - indexing as shown within each $P$-slice, then incrementing the ABS index and repeating. In this way, only the non-vanishing blocks need be stored, but these can still be rapidly accessed individually due to the redundant, zero-sized matrices. As such, sparsity is not accurately reflected within each block. However, the number of significant blocks per fragment will eventually reach a fixed size, such that the storage and evaluation of the integrals reaches linear scaling. The data redundancy is a small compromise that allows 
for a considerably faster Fock build routine, as will be discussed shortly.

An estimate for the maximum value within a block, weighted by the initial density $\mathbf{P}$, can be found using the Cauchy-Schwarz inequality ${ }^{118}$ and the distance dependence in Equation 24:

$$
\left\|\mathbf{B}_{X Y}^{Z} \mathbf{P}^{X Y}\right\|_{\infty} \lesssim 2\left\|\mathbf{g}_{X Y X Y}\right\|_{\infty}^{1 / 2}\left\|\mathbf{G}_{Z Z}\right\|_{\infty}^{1 / 2}\left\|\mathbf{P}^{X Y}\right\|_{\infty} R_{(X Y) Z}^{-1}
$$

where $R_{(X Y) Z}$ is the distance from fragment $Z$ to the midpoint of fragments $X$ and $Y$. This requires an accurate initial density, and evaluation of the diagonal two-electron integrals, $\mathbf{g}_{X Y X Y}$. The latter can very efficiently be computed and stored once at the beginning of the calculation, and the density is formed from the converged monomer densities, such that it should be a good initial guess. Using this coarse, fragment-blocked screening, the integral evaluation and storage becomes linear scaling in system size. However, the Cholesky decomposition of the two-index quantities is still necessary, and eventually for very large systems, this will dominate the cost.

\subsection{Local Fock build}

The two-electron integrals and density matrix are used in each iteration of the SCF procedure to form the Fock matrix. The core Hamiltonian part is inexpensive - it is the Coulomb and exchange portions that conventionally show quartic dependence on the number of basis functions. Of these, the latter is in principle much more complicated than the former. Within the density fitting framework, the exchange matrix can be written as

$$
K_{X \mu, Y \nu}=\sum_{W, Z}\left\langle\mu^{X} \tau^{W}\left|r_{12}^{-1}\right| \lambda^{Z} \nu^{Y}\right\rangle P^{W \tau, Z \lambda} \approx \sum_{W, Z} b_{X \mu, W \tau}^{Q} b_{Z \lambda, Y \nu}^{Q} P^{W \tau, Z \lambda}
$$

The contraction with the density occurs across the two three-index integral tensors, resulting in an apparent quintic cost. However, if we write the density in the symmetrically 
orthogonalized basis, we get

$$
K_{X \mu, Y \nu} \approx\left(\sum_{W} b_{X \mu, W \tau}^{Q} \tilde{T}_{\cdot i}^{W \tau}\right)\left(\sum_{Z} b_{Y \nu, Z \lambda}^{Q} \tilde{T}_{\cdot i}^{Z \lambda \cdot}\right)=\tilde{k}_{X \mu}^{i, Q} \tilde{k}_{\cdot Y \nu}^{Q, i}
$$

which is cubic scaling.

However, a cubic scaling term every iteration is still a problem. Exchange is inherently a short-range effect, such that we can use the inherent localization of the ALMOs to reduce the unphysical cost. A 'local exchange' approach was first proposed by Polly and coworkers, ${ }^{31}$ and has more recently been improved by Köppl and Werner; ${ }^{32}$ we use their method as a starting point. The basic idea is that each localized orbital only has significant overlap with other orbitals within a fixed extent. Therefore, for each molecular orbital, $i$, an orbital domain can be assigned, $[i]$, comprising the atomic orbitals that will give non-vanishing contributions to the exchange. As the system size increases, the domain sizes eventually become fixed due to the localization, such that the calculation becomes linear scaling.

The use of density fitting requires that three such domains be defined per occupied orbital: a local MO domain, $[i]_{\mathrm{MO}}$; an $\mathrm{AO}$ domain, $[i]_{\mathrm{AO}}$; and an auxiliary domain, $[i]_{\mathrm{ABS}}$. This can be seen by partitioning Equation 26:

$$
\begin{aligned}
\tilde{k}_{X \mu \cdot}^{i, Q} & =\sum_{W \tau \in[i]_{\mathrm{MO}}} b_{X \mu, W \tau}^{Q} \tilde{T}_{\cdot i}^{W \tau}, X \mu \in[i]_{\mathrm{AO}}, Q \in[i]_{\mathrm{ABS}} \\
K_{X \mu, Y \nu} & \approx \sum_{i} \sum_{Q \in[i]_{\mathrm{ABS}}} \tilde{k}_{X \mu}^{i, Q} \tilde{k}_{\cdot Y \nu}^{Q, i}, X \mu, Y \nu \in[i]_{\mathrm{AO}}
\end{aligned}
$$

A clear disadvantage of this approach in general is that the results so obtained are likely to be highly sensitive to the choice of domains. This is where the fragment localization of the present method is particularly useful. Instead of considering the significance of individual orbitals, the contribution of a fragment as a whole can be considered. This will of course result in the inclusion of orbitals that are not necessary, but the well separated nature of the fragments precludes the exclusion of important contributions, somewhat resolving the 
strong dependence on choice of domain.

We use the following criteria for the domains. The AOs of fragment $X$ are included in $[i]_{\mathrm{MO}}$ if they make a significant contribution to the density on $i$; that is, if

$$
\rho_{X \rightarrow i}=\sum_{\mu \in X} \tilde{T}_{\cdot \mu}^{i \cdot} \tilde{T}_{\mu \cdot}^{\cdot i}>\epsilon_{\mathrm{MO}}
$$

where $\epsilon_{\mathrm{MO}}$ is a user-defined threshold. Typically a value of $\epsilon_{\mathrm{MO}} \approx 10^{-6}$ suffices. It is then presumed that, almost by definition, if the density of $i$ has a significant contribution from $\{X \mu\}$, then this will also be true of $\tilde{k}$. Thus $[i]_{\mathrm{MO}} \subset[i]_{\mathrm{AO}}$. However, as the Coulomb force decays slowly with separation, the AO domain will in general be considerably larger than the MO domain. Let $\mu$ denote the exponent of the most diffuse basis function on $X$, and assume that the extent of this function - the cutoff beyond which its norm changes less than a given threshold, $\epsilon_{\mathrm{AO}}$ - is much greater than one. Then, for $i$ located on fragment $Y$, we include all the atomic orbitals from fragment $X$ if

$$
r_{X} \approx\left[\frac{\ln (\sqrt{2 \pi})-\ln \left(\mu \epsilon_{\mathrm{AO}}\right)}{2 \mu}\right]^{1 / 2}<R_{X Y}
$$

where $R_{X Y}$ is the center-of-mass separation of the two fragments. Again, a reasonable threshold is $\epsilon_{\mathrm{AO}}=10^{-6}$. Finally, the ABS domains are found by a population analysis, as suggested by Polly et al. ${ }^{31}$ For $i$ on $Y$, the ABS of $X$ is included in $[i]_{\text {ABS }}$ if the partial charge associated with charge transfer from $i$ to $X$ is greater than a threshold, $\epsilon_{\mathrm{ABS}}$. However, for ALMOs, a Mulliken analysis gives zero charge transfer, such that a Löwdin analysis must be used instead. The condition is thus:

$$
q_{i \rightarrow X}=\sum_{\mu \in X}\left[\mathbf{S}^{1 / 2} \tilde{\mathbf{T}}\right]_{\mu i}^{2}>\epsilon_{\mathrm{ABS}}
$$

In the original local exchange method, ${ }^{31,32}$ the domain would be further extended by including all atoms connected to the atom of the current orbital. However, by assumption, there 
are no covalent bonds between fragments here, such that this is not necessary.

In general, the domains should need to change with each iteration. However, given a high quality initial density, such as is provided by the converged monomer densities, the domains can be fixed in the first iteration. An additional complication, though, is that the restriction of the fitting basis means that the fitting metric, $\mathbf{G}$, is no longer valid in the restricted space. As such, the full metric needs to be restricted by retaining only the blocks included in the ABS domain, but this needs to be done separately for each occupied orbital. In practice, this is a minor technical issue that does not affect the efficiency of the algorithm.

At face value, the Coulomb contribution to the Fock matrix should be much simpler. Using density fitting, it is given by

$$
J_{\mu \nu}=b_{\mu \nu}^{Q}\left[\mathbf{b}^{T} \mathbf{P}\right]_{Q}
$$

meaning it can be formed by two $\mathcal{O}\left(N^{2} M\right)$ matrix-matrix products. It is tempting to try to reduce this cubic scaling in the same manner as for the exchange, but as has already been mentioned, the Coulomb interaction falls off much more slowly with distance. As such, prohibitively large domains would be needed. Moreover, the local exchange algorithm requires storage of the raw three-center integrals, rather than those symmetrised with the inverse fitting metric. Therefore, we instead form the Coulomb matrix as $\left(\mu^{X} \nu^{Y} \mid P^{Z}\right) \tilde{B}_{Z P}$, where $\tilde{\mathbf{B}}=\mathbf{G}^{-1} \mathbf{P}^{X Y}(X Y \mid Z)$. In this way, we need only loop over nonzero integral blocks, $(X Y \mid Z)$, something which is also true for Equation 27 with the raw integrals. Thus the integral blocking not only severely reduces the memory imprint, but also more rapidly brings the computational cost of the Fock build to linear scaling. The only remaining non-linear term is the multiplication by the inverse metric in the Coulomb part, which is quadratic. Note that, while $\mathbf{G}$ will become sparse in the macroscopic limit, this does not imply its inverse will be sparse - in fact, the opposite is usually true. ${ }^{119}$ However, as this is a single matrix-matrix multiplication, it is a negligible cost. 


\subsection{Pairwise RPAd}

The calculation of the RPA dispersion energy requires integrals transformed to the molecular orbital basis, which can be achieved using density fitting as described earlier. Note that the requirements on the auxiliary basis to minimize DF errors are different for the integral transformation compared to the Fock build, so in general a separate ABS must be used. For direct RPA and RPA+SOSEX we have that $\mathbf{K}=\tilde{\mathbf{b}} \tilde{\mathbf{b}}^{T}$, allowing us to avoid ever forming the full four-index integral tensor. Moreover, we can use this to rewrite Equation 16 as

$$
\tilde{\epsilon} \tilde{\mathbf{t}}+\tilde{\mathbf{t}} \tilde{\epsilon}=-\mathbf{u u}^{T}
$$

where $\mathbf{u}=(1+\tilde{\mathbf{t}}) \tilde{\mathbf{b}}$. This is not possible for RPAx due to the exchange terms in the residual. The most expensive computation in each iteration will thus be the evaluation of the quadratic term on the right hand side, which is $\mathcal{O}\left(O^{2} V^{2} M\right)$ for dRPA and $\mathcal{O}\left(O^{3} V^{3}\right)$ for RPAx. Thus these are formally fifth- and sixth-order scalings with system size, although the number of occupied orbitals, $O$, is generally much smaller than $N$.

As several iterations are required, this scaling is prohibitive. However, the fragmentbased localization again allows for reduction to linear scaling. In the energy decomposition, we partitioned the RPA excitations as per Figure 1, dispensing with all but the dispersive terms, where such terms are inherently limited to pairs of fragments as rCCD (and therefore RPA) is restricted to double excitations. This does not mean that only two-body effects are included, though, as RPA implicitly includes contributions to infinite order at the orbital level. However, it does allow us to adopt a natural local correlation approach, where the orbital domains are restricted by performing separate RPA calculations on pairs of fragments. If the ALMO orbitals were used directly, this would be exact, but the projection of the virtuals makes the pairwise approach an approximation. On the other hand, one only needs to project the virtuals out of the smaller occupied space for the pair, such that localization is better maintained. Moreover, as dispersion has an asymptotic $R^{-6}$ dependence, only pairs 
within a given distance of each other (we measure the center-of-mass separation) need be included, such that the number of RPA calculations per fragment eventually becomes fixed, leading to linear scaling. We term this approach 'pairwise RPAd', where the ' $d$ ' denotes the restriction to dispersion and exchange-dispersion excitations.

\subsection{Parallelization}

The fragment-based partitioning of the monomer calculations, integrals, Fock build, diagonalizations, and pairwise RPA calculations make this method almost embarrassingly parallelizable. The lack of significant communication required in all but the formation of the density ideally suits a distributed memory parallelism, and several portions of the method would benefit from the use of accelerators. The technical hurdles involved with such massive parallelism are beyond the scope of this paper. Instead, we consider a simple multithreaded approach that can later be combined with a larger scale parallelization.

Firstly, all integrals can trivially be threaded by balancing over shell pairs, triplets, or quartets, depending on the integral class. This can be done separately within each fragment integral block, such that the blocks can themselves later be parallelized over. Assuming that the orbital domains are of roughly similar sizes on each fragment - a coarse but reasonable assumption - each fragment block of the Fock build can be threaded by chunking up the largest domain (always the AO domain). This is particularly simple for the exchange portion, but for the Coulomb portion the first half transformation of the density-fitted integrals must be completed on all threads before the second half transformation is performed, somewhat limiting the effectiveness of the multithreading. Instead for this portion it would perhaps be better to multithread the matrix multiplications. In the core ALMO iterations, the formation of the density matrix and inverse metric necessitates shared memory, such that these parts can simply be threaded fragment-by-fragment. The individual fragment diagonalizations can also make use of standard multithreaded linear algebra routines, as can the contractions in the iterative solution of the RPA amplitude equations. 


\subsection{Summary}

Table 1: Summary of the formal scaling of the different parts of the ALMO+RPAd calculation, along with their conventional counterparts. Upper and lower case quantities refer to the system and fragment sizes, respectively.

\begin{tabular}{lccc}
\hline Procedure & Overall & Fragment & Conventional \\
\hline Full ERIs & - & $n^{4}$ & $N^{4}$ \\
DF ERIs & $F n^{2} m$ & $n^{2} m$ & $N^{2} M$ \\
Cholesky & - & - & $M^{3}$ \\
Diagonalisation & $F n^{3}$ & $n^{3}$ & $N^{3}$ \\
Coulomb & $F M n^{2}$ & - & $N^{4}$ \\
Exchange & $F n^{4}$ & $n^{4}$ & $N^{4}$ \\
Charge transfer & $V^{2}$ & $v^{2}$ & - \\
RPA & $F o^{3} v^{3}$ & $o^{3} v^{3}$ & $O^{3} V^{3}$ \\
\hline
\end{tabular}

The scalings of the different components of the calculation with respect to both fragment and system size are given in Table 1. It shows that all of the major steps are essentially linear scaling, with the exception of the Coulomb part of the Fock matrix. This is, however, only formally quadratic due to an unavoidable matrix-matrix multiplication; properly accounting for sparsity would eventually (but slowly) lead to linearity. The density fitting, however, requires a cubic Cholesky decomposition, which will dominate for very large systems, despite the efficiency of standard algorithms. We are currently looking in to how this can be circumvented. Finally, the on-fragment scaling is still dominated by the RPA calculation. In principle, this could be alleviated by further use of local correlation methods, but applying such an approach would require a very careful consideration of the additional errors it would introduce.

\section{Results}

The ALMO+RPA method has been implemented in our in-house electronic structure package, ${ }^{120}$ which utilises the LIBINT package ${ }^{121}$ of Valeev and coworkers for the molecular integrals, and the EIGEN library ${ }^{122}$ for all linear algebra, including tensor manipulations. Ad- 
ditional DF-MP2, ${ }^{34}$ CCSD, ${ }^{123}$ CCSD(T), ${ }^{123,124}$ and M06-2X ${ }^{125}$ calculations were performed in the MOLPRO package, ${ }^{126}$ while DF-SAPT ${ }^{66,127}$ calculations were carried out using Psi4. ${ }^{128}$ Unless noted otherwise, all calculations use the aug-cc-pVDZ (aVDZ) orbital basis ${ }^{129-131}$ with the associated JKFit ${ }^{30,132,133}$ and MP2Fit ${ }^{134}$ density fitting sets for the Fock and MO-basis integrals, respectively. All timing and memory benchmarks are reported for calculations on a single processor on the same compute node, with two 10-core Intel ${ }^{\circledR}$ Xeon ${ }^{\circledR}$ CPUs and 256 GB of RAM. Scalings with system size are reported for the RPAx variant of the method, as this is the most computationally complex variant, as discussed earlier. In the following, we apply the ALMO+RPA method to a variety of different systems, including benchmarking against the S66 database. ${ }^{10,135}$ In this way, we demonstrate and assess the accuracy of the method, the errors associated with the various approximations, and the scaling with both fragment and system size. Finally, we compare the energy decomposition with that from symmetry-adapted perturbation theory.

\subsection{Scaling}

The scaling with number of basis functions per fragment is demonstrated for the water dimer in Figure 3, by using the progression of orbitals basis sets: cc-pVnZ and aug-cc-pVnZ, with $\mathrm{n}=\mathrm{D}, \mathrm{T}, \mathrm{Q} .{ }^{129,130}$ The ALMO+RPAxd curve has a scaling exponent of 2.7, or approximately cubic, as should be expected from Table 1. Moreover, this represents savings of several orders of magnitude over conventional counterpoise-corrected CCSD calculations, as can be seen from the Figure.

To study the scaling with overall system size, we consider two extreme cases: a linear chain of hydrogen fluoride molecules, and a series of water clusters. The former corresponds to the best possible scenario, with a single hydrogen bond added per new fragment, and with interactions along a single spatial axis. The clusters, on the other hand, are known to be particularly difficult, ${ }^{136}$ as they are tightly bound, with multiple interactions per fragment. Firstly, the efficacy of the fragment-blocked density fitting scheme is shown in Figure 4. In 


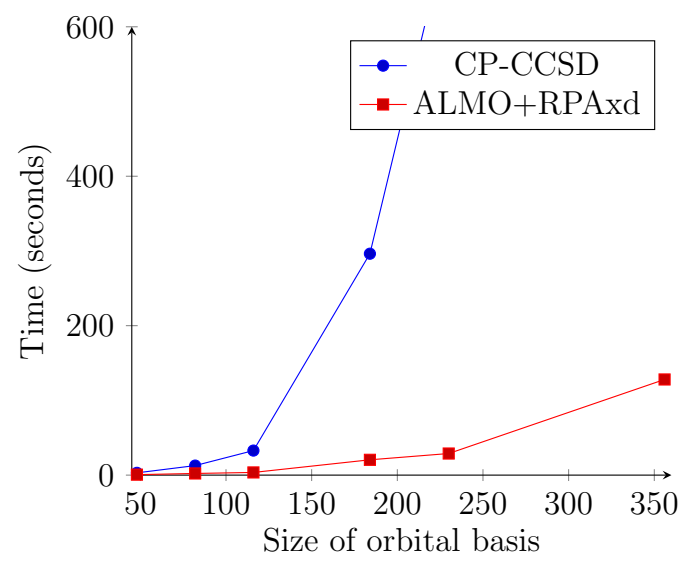

Figure 3: The total time taken to calculate the interaction energy for the water dimer in basis sets of increasing size. The overall scaling for ALMO+RPAxd is cubic, compared to the sixth-order scaling of CCSD, leading to substantial speedups.
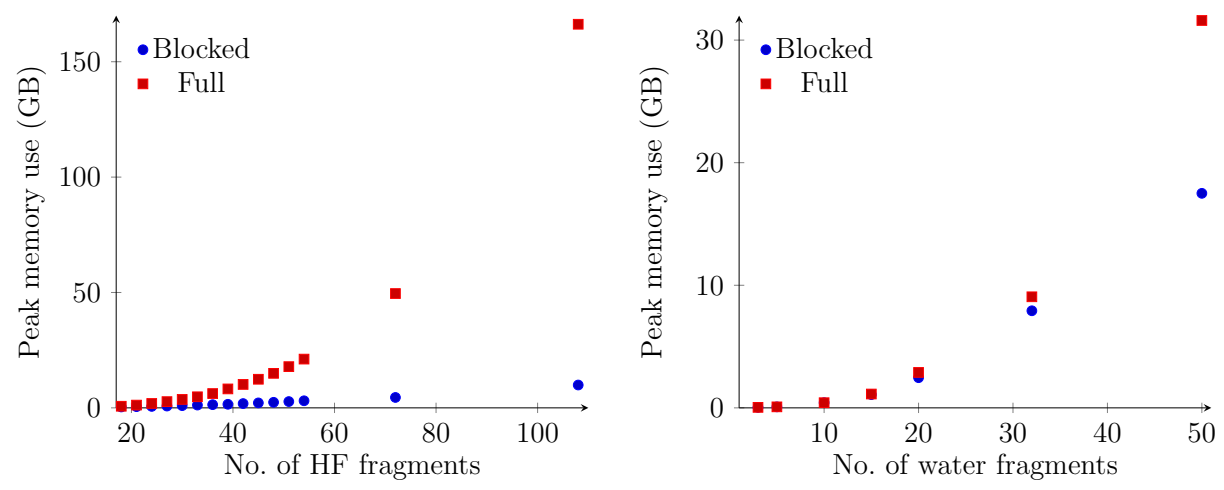

Figure 4: The peak amount of memory required during the ALMO+RPAxd calculation on either the linear hydrogen fluoride chains (left) or water clusters (right), showing the improvements resulting from using the blocked integrals as opposed to the conventional density fitting scheme. 
both cases, the memory requirements become linear, but this happens much more rapidly for the chain. The reduction in memory means that 108 hydrogen-fluoride molecules (roughly 3500 and 12500 orbital and auxiliary basis functions) or 50 water molecules (2000 and 7500 functions) can be considered entirely in-core with slightly over 16 GB of memory. The different rates at which these savings are reached is due to the different sparsities of the underlying integral tensors,

The time spent on each portion of an ALMO+RPAxd calculation as a function of total system size is shown in Figure 5. From this, the linear scaling is clear to see, with the
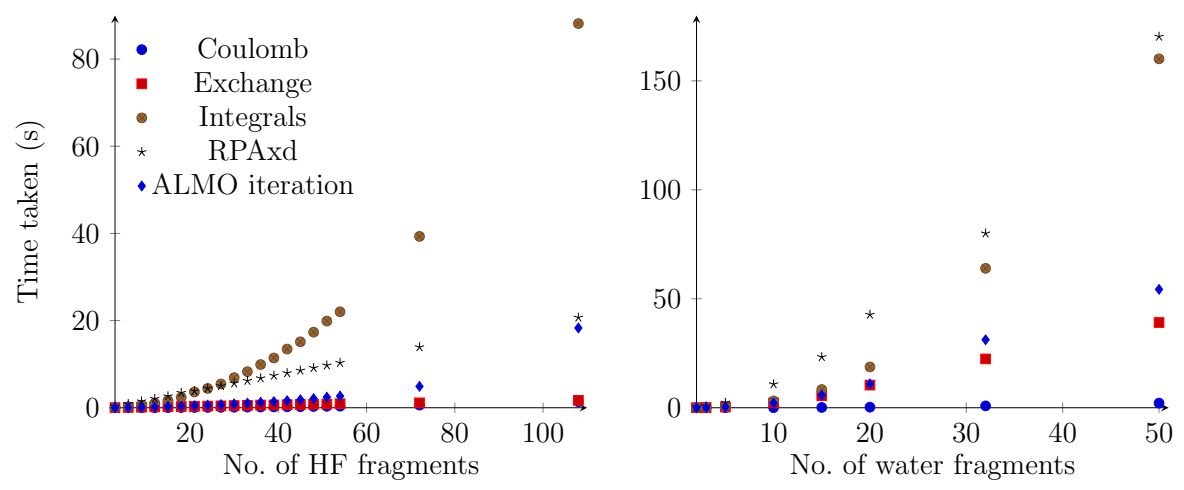

Figure 5: The amount of time spent in each part of the ALMO+RPAxd calculation, for varying sizes of $\mathrm{HF}$ chains (left) and water clusters (right). The Coulomb and exchange timings are per iteration, while the integral timings include the Cholesky decomposition required to form the inverse fitting metric.

exception of the integrals. In fact, the integral routines themselves are linear, and this is a significant contributor to the cheapness of the Coulomb and exchange terms; instead it is the Cholesky decomposition of the fitting metric hidden in this term that is leading to unfavourable scaling. In fact, for the 108 hydrogen fluoride fragments, just the Cholesky routine takes up roughly $40 \%$ of the total computation time. For larger fragments, however, it will take longer for this to become the bottleneck. Nevertheless, it would be best to try to avoid this problem, and attempts have been made in this direction elsewhere. ${ }^{137}$ We also note that the Coulomb term should not formally be linear, but appears to be so here, most likely by virtue of how inexpensive it is overall.

The significant reduction in cost is due to a number of approximations, each of which 
has an associated error. Figure 6 reports the average error in the interaction energy per fragment due to the density local and pairwise RPA approximations for the first few fluoride chains. This shows that the error due to both, when the thresholds stated earlier are used, is less than $0.1 \mathrm{kcal} \mathrm{mol}^{-1}$ in the total interaction energy, and stays remarkably static as the system size increases. In particular, the change in the density due to using the local approximation has essentially no effect on the correlated part of the calculation, and the loss of three-body effects in the pairwise RPA is insignificant. Additional errors, and calibration graphs for the various thresholds, are given in the supporting information.

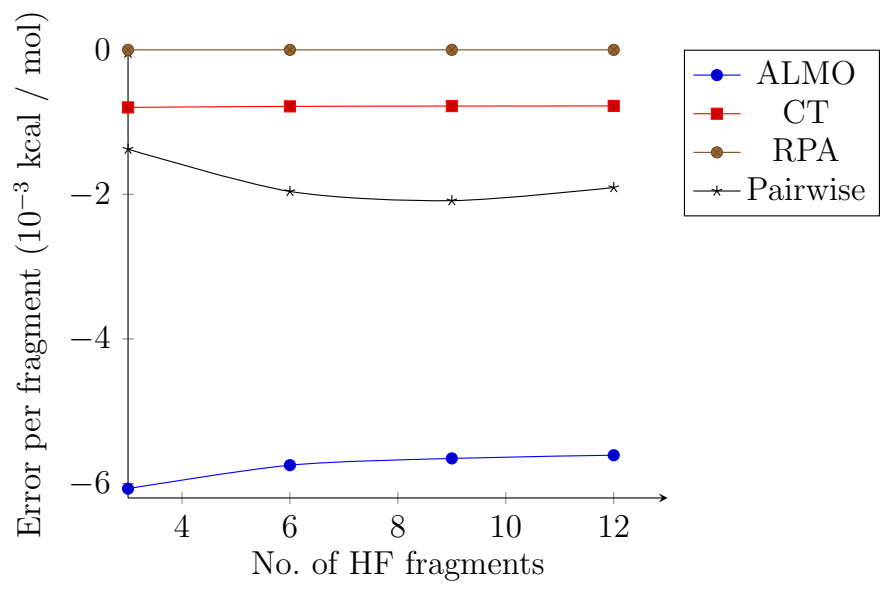

Figure 6: The error per fragment in the different contributions to the interaction energy caused by using the local approximation in the Fock build and the pairwise approximation in the RPAxd calculation, for different sizes of hydrogen fluoride chains.

Finally, the performance of the multithreading described earlier is shown for both a large chain and a large water cluster in Figure 7. This shows excellent speedups up to around four threads, before starting to tail off. The effect is much larger in the water cluster, as this is dominated by the parallelized bits of code, in particular the Fock build. The fluoride chain, on the other hand, is dominated by the Cholesky decomposition, which was not threaded here. Overall, using only eight threads, the calculation on a cluster of 32 water molecules can be carried out in under three minutes; when combined with a distributed, fragment-based parallelism, there is clearly potential for very large systems to be considered with minimal time requirements. 


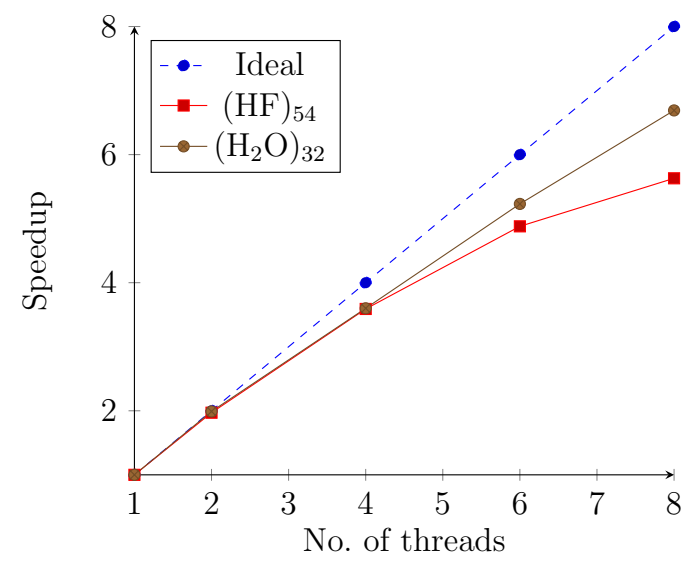

Figure 7: The combined speedup in computation time for the integral evaluation, Fock build, and RPA portions of the calculation using multithreading. Results are given for the $(\mathrm{HF})_{54}$ chain and $\left(\mathrm{H}_{2} \mathrm{O}\right)_{32}$ cluster. The calculations were performed on the same compute node, separately, with no other calculations running. The timings for the serial calculations were 3.3 and 18.1 minutes for the fluoride chain and water cluster, respectively.

\subsection{Potential energy curves}

A fast, linear-scaling method is useless if the results are not accurate. As non-covalent interactions cover such a broad remit, it is important to test the method on a wide range of examples. Moreover, it is important to go beyond a single interaction energy - the method should also accurately reproduce equilibrium geometries, and molecular properties. We demonstrate this by considering potential energy curves for four different bimolecular complexes, as a function of the intermolecular separation. These systems are: the water dimer, a widely studied example of hydrogen bonding; $\mathrm{FCl} \cdots \mathrm{OH}_{2}$, an unusually strong halogen bond; ${ }^{138}$ the helium-neon complex, which is purely dispersively bound; and the complex of benzene and $\mathrm{HCN}$, where the cyanide is perpendicular to the benzene ring. Geometries are given in the supporting information.

Figure 8 compares the ALMO+RPA(SOSEX)d results with other double excitation theories, namely counterpoise-corrected CCSD and MP2. In addition, the CCSD result without counterpoise correction is given, demonstrating that the BSSE has been successfully eliminated. In all cases, the new method is in excellent agreement with the coupled cluster results, while MP2 performs considerably worse. Reassuringly, the locations of the energy minima 

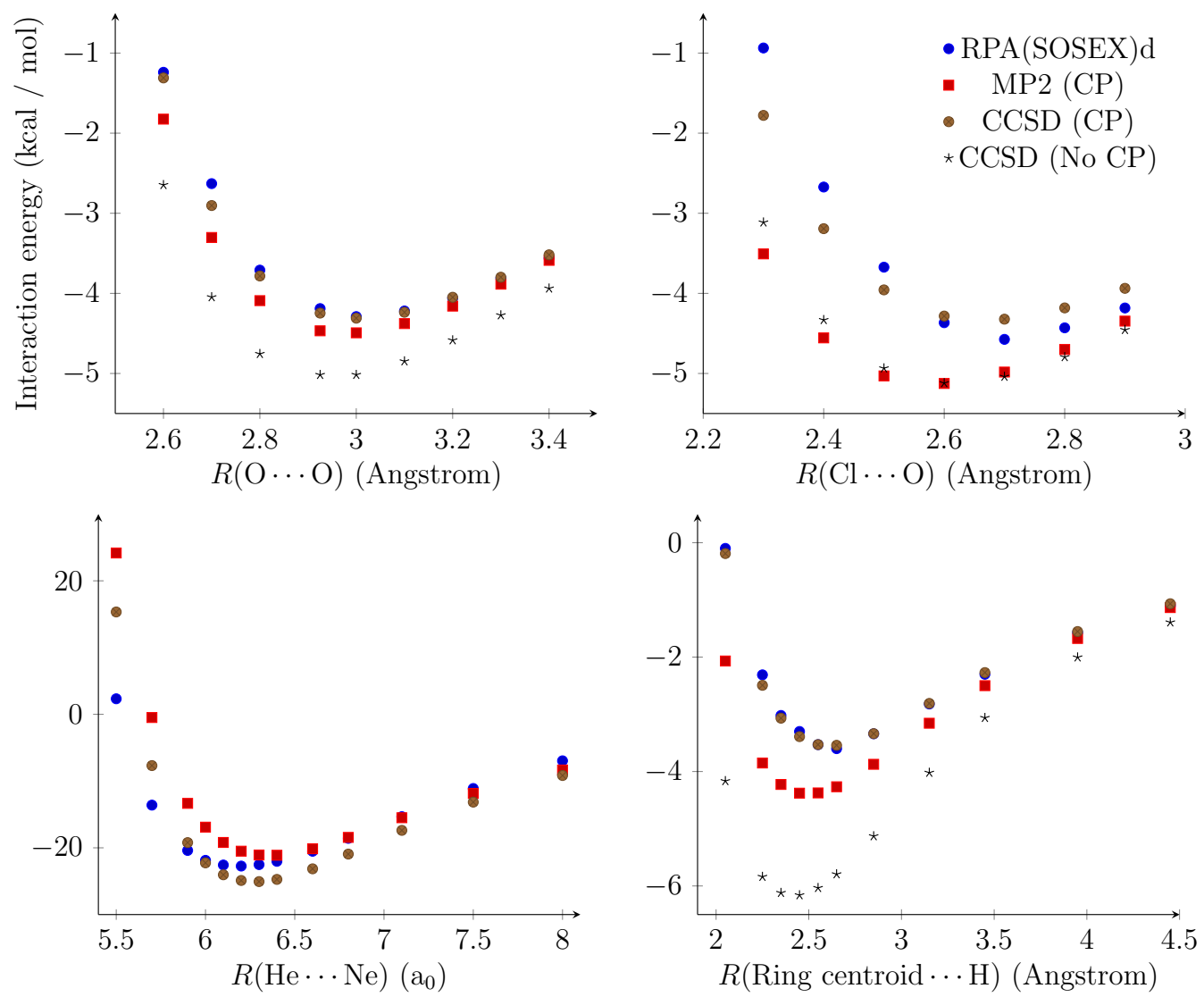

Figure 8: Potential energy curves for the water dimer (top left), $\mathrm{FCl} \cdots \mathrm{OH}_{2}$ (top right), He $\cdots$ Ne (bottom left), and benzene with HCN (bottom right). The CCSD without counterpoise is not included for the helium-neon complex, as the error is so large it makes it impossible to see the differences in the other curves. 
and the overall shapes of the curves are reproduced by the new method.

\subsection{Performance on the S66 benchmark set}

We have performed more extensive benchmarks on single-point calculations at equilibrium geometries, by doing so for every complex in the S66 database of biologically relevant noncovalent interactions. ${ }^{135}$ These are of particular interest, because one of the most appealing uses of the new method is to investigate how solvent affects the interactions in such systems. Calculations were performed using a variety of different methods with the aVDZ basis sets, with geometries (see SI) optimized at the CCSD/aVDZ level. The database is split primarily into three different 'types' of interaction: hydrogen bonded, dispersively bound, and other (usually a mixture of the two). It is also possible to instead split by the dominant component in the SAPT decomposition: electrostatics, dispersion, or an even mix of the two; we will compare the classifications from the new method's energy decomposition to those from SAPT in the next section.

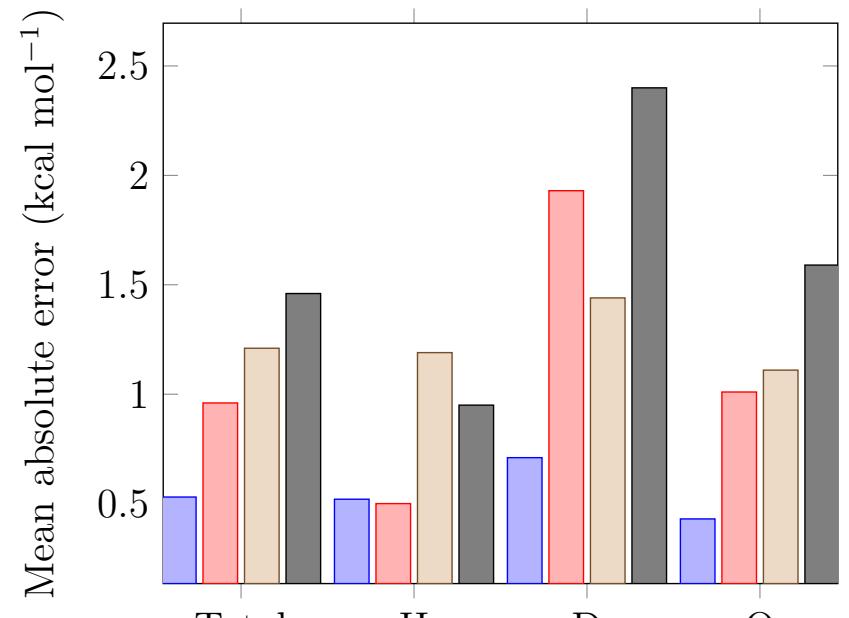

$$
\begin{aligned}
& \text { Total H D O }
\end{aligned}
$$

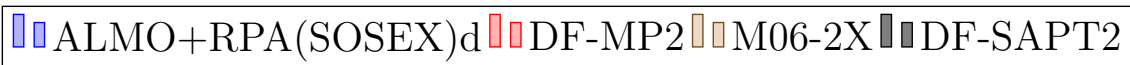

Figure 9: The mean absolute errors for different methods with an aVDZ basis, across the S66 database, relative to the CCSD/aVDZ results. The errors are further split by interaction type, as in the original S66 paper: hydrogen-bonded $(\mathrm{H})$; dispersion dominated (D); and other $(\mathrm{O})$. 
In Figure 9 we see that the ALMO+RPA(SOSEX)d method gives far better agreement with the equivalent CCSD results than any of the other methods, in particular in the case of the dispersion-dominated complexes. The dRPA and RPAx variants show similar but slightly worse errors, as they under- and over-predict the exchange contribution, respectively; all raw results are given in the supporting information. It should be noted that, as MP2 tends to overbind the electrostatically-dominated systems, it can often appear closer to $\operatorname{CCSD}(\mathrm{T})$ results than CCSD, for small basis sets. However, this is a fortunate error correction, rather than a physical feature of MP2 - it does not contain the physics described by a triples correction. Moreover, as it overbinds dispersive complexes, the improvement is not consistent across all systems, so cannot be relied upon.

It is useful to try to disentangle the error due to the use of ALMOs from that of retaining only the ring coupled cluster diagrams. This can be done by comparing the ALMO energies to counterpoise-corrected Hartree-Fock energies. Without the charge transfer correction, the mean-absolute error across the whole set is $0.95 \mathrm{kcal} \mathrm{mol}^{-1}$, reducing to $0.33 \mathrm{kcal} \mathrm{mol}^{-1}$ when the full, iterative correction in Equation 12 is applied. The latter error stays fairly consistent across all classes of interaction, at $0.25,0.42$, and $0.32 \mathrm{kcal} \mathrm{mol}^{-1}$ for the hydrogen bond, dispersion, and other categories, respectively. The average BSSE present in the HF and CCSD correlation energies is -0.65 and $-1.39 \mathrm{kcal} \mathrm{mol}^{-1}$, showing this has been effectively eliminated.

There is a technical issue here with the use of the local exchange procedure, however. As noted by Köppl et al., ${ }^{32}$ while the density is fairly insensitive to the local approximation, the energy is often quite sensitive to the choice of domains. As such, they recommend performing a single, full exchange build from the converged density; the difference between this and the local result will here be termed the X-correction. The coarseness of the domain building, i.e. its fragment-based nature, means however that the energy is far less sensitive in most cases. In fact, for all but the dispersion-type systems, it accounts for less than $3 \%$ of the total interaction energy. However, unless the distance threshold in the domain selection is 
adjusted to reflect the longer separations in the dispersive complexes, the X-correction can account in magnitude for up to $50 \%$ of the total interaction, and on average $9 \%$, and so must be included. The larger percentage is also partly due to the fact that these systems tend to have smaller interaction energies. This may become a problem when more fragments are present, as the full exchange build will scale cubically with system size; in these cases it is then best to select a distance cutoff where at least nearest-neighbour fragments are included in the domains for a fragment.

Table 2: Ratios of total wall time for various methods to that for ALMO+RPAxd, with and without the local exchange correction. Ratios are averaged across the S66 test set, with calculations carried out on a single core (32 GB of memory).

\begin{tabular}{lrrrrr}
\hline & DF-MP2 & CCSD & CCSD(T) & M06-2X & DF-SAPT2 \\
\hline With X-correct & 2.2 & 21.7 & 76.5 & 2.0 & 4.9 \\
Without X-correct & 2.9 & 27.9 & 99.6 & 2.5 & 6.4 \\
\hline
\end{tabular}

The average timings for DF-MP2, CCSD, CCSD(T), M06-2X and DF-SAPT2 relative to ALMO+RPAxd both with and without the X-correction are given in Table 2. These show that even with the correction term, the new method is at least twice as fast as density-fitted MP2, and over an order of magnitude faster than CCSD, despite giving very similar results to the latter. Moreover, these speedups will increase rapidly when either the number of fragments or size of the basis is increased. To consider convergence of the energies with respect to the latter, calculations were performed on the benchmark geometries from the original S66 paper using the aVTZ basis sets; the mean-absolute error compared to the complete basis set limit CCSD results was $0.60 \mathrm{kcal} \mathrm{mol}^{-1}$.

\subsection{Comparison with SAPT}

In the above, DF-SAPT2 was chosen for comparison as it is probably the most similar method currently available to the ALMO+RPA approach: it eliminates BSSE from the beginning, it is designed specifically for interaction energies, it contains a coupled cluster-style correlation 
energy, and it provides an energy decomposition analysis. The components from both the present method and SAPT for the entire S66 dataset using the aVTZ basis are given in the supporting information. Here, we will just compare how the new method classifies the different interactions, and thus whether this agrees qualitatively with SAPT.

The original classification came from the ratio of dispersion to electrostatic energy terms. ${ }^{135}$ However, there is no direct comparison of the SAPT electrostatic term with, for example, the frozen energy term in the ALMO EDA, because the latter already includes exchange. Instead, we note that by calculating the SAPT charge transfer term of Stone and Misquitta, ${ }^{139}$ we can extract an equivalent to the ALMO polarization term by removing charge transfer from the SAPT induction. Then considering the ratio, $r_{\mathrm{D} / \mathrm{P}}$, of dispersion to polarization, we can recover exactly the original classifications: electrostatic, $r_{\mathrm{D} / \mathrm{P}}<2$; mixed, $r_{\mathrm{D} / \mathrm{P}}<8$; dispersive, $r_{\mathrm{D} / \mathrm{P}} \geq 8$. A comparison is shown in Figure 10. In general it can be seen that qualitative agreement between the two is good, with only four complexes resulting in different categories being assigned. Two of these are borderline cases where one method gives $r_{\mathrm{D} / \mathrm{P}} \approx 7.9$ and the other slightly over the cutoff (T-shaped benzene dimer and benzene... $\mathrm{MeNH}_{2}$ complexes). The other two $\left(\mathrm{MeNH}_{2}\right.$ with peptide and pyridine) the ALMO EDA classifies as electrostatic $\left(r_{\mathrm{D} / \mathrm{P}} \approx 1.6,1.8\right)$ whereas SAPT classifies them as mixed $\left(r_{\mathrm{D} / \mathrm{P}} \approx 3.0,5.6\right)$. In both instances, this is due to SAPT predicting a larger dispersion contribution than ALMO+RPA. This is borne out in the total interaction energies, where SAPT and ALMO+RPA over- and underbind by about $0.5 \mathrm{kcal} \mathrm{mol}^{-1}$, respectively, compared to CCSD/CBS results.

\section{Conclusions}

We have presented a new method capable of accurately determining BSSE-free interaction energies for supramolecular systems composed of many fragments. By building on absolutely localised molecular orbitals and applying a variety of cutting-edge computational chemistry 


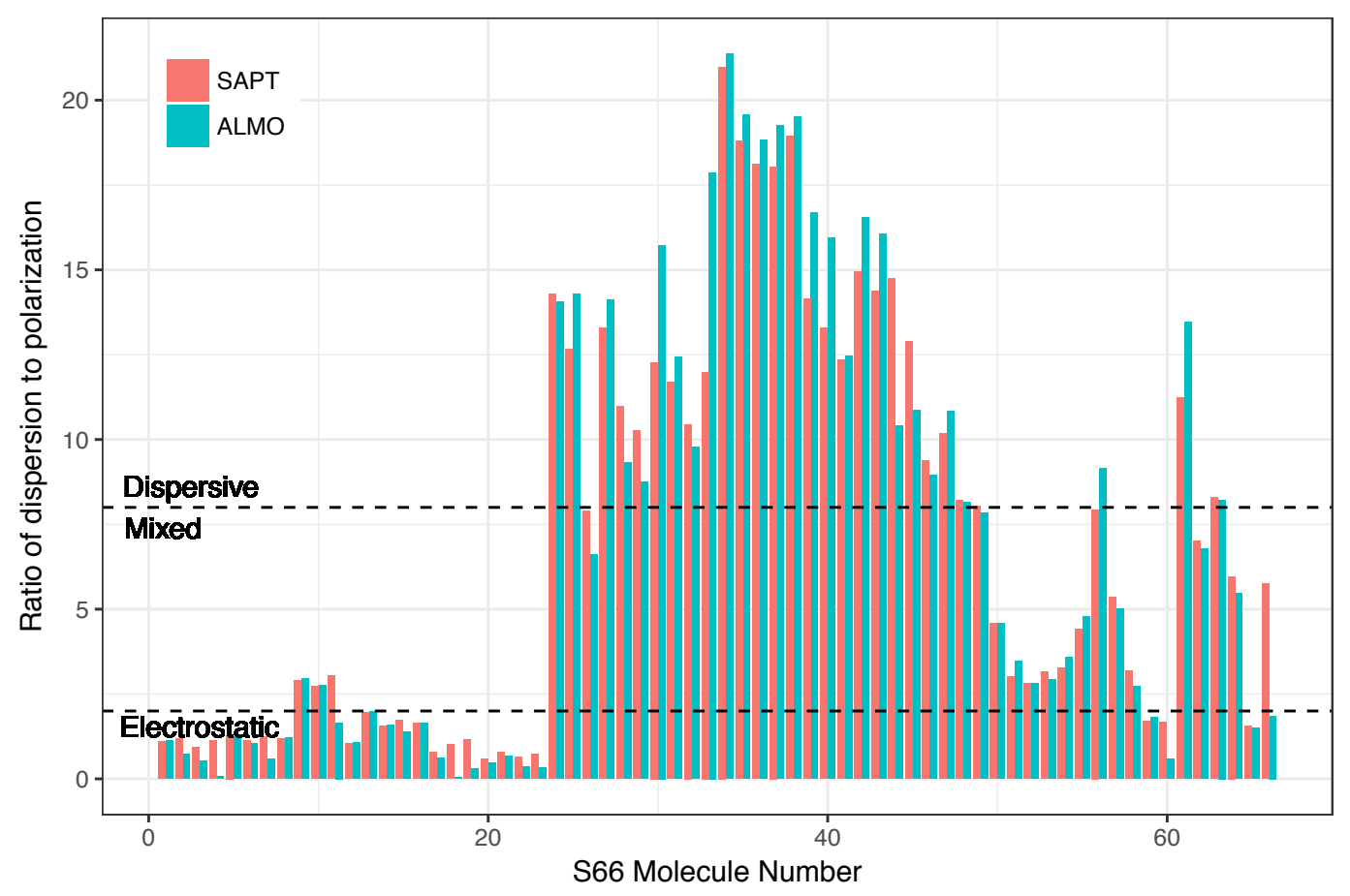

Figure 10: The ratio of dispersion to polarization contributions, $r_{\mathrm{D} / \mathrm{P}}$, in the interaction energies of the complexes in the S66 database. Results are compared for the SAPT (red, left bar) and ALMO (blue, right bar) energy decompositions, with the cutoffs for classification shown as dashed lines. The molecule number is as given in the original database. ${ }^{135}$ All numerical data can be found in the supporting information. 
techniques, it has been possible to arrive at a procedure that gives CCSD quality results for a range of chemically interesting systems at a fraction of the cost of full, counterpoise-corrected calculations. In particular, a chain of 108 hydrogen fluoride molecules - corresponding to over 1000 electrons, 3500 orbital basis functions, and 12500 auxiliary basis functions - could be treated on a single processor in 486 seconds, or slightly over eight minutes, using less than ten gigabytes of memory. It was found that by specialising to systems composed of a large number of small- to medium-sized monomers, it is possible to make full use of the inherent localisation of the ALMOs both in the mean-field and correlated regimes. In particular, problems with large energy errors in the local Fock build are alleviated by using a coarser definition of the orbital domains, and large superposition errors are mostly removed from the correlation energy by decomposing the excitations into distinct terms. Finally, the new method allows the interaction energy to be decomposed into physically distinct terms in a quantitatively accurate way. This coupled with the essentially linear-scaling and inherently parallel nature of the calculation, opens up the possibility of gaining insight into the interactions in extended systems, such as polymers, supramolecular complexes, and even crystals.

There are caveats, however, mainly in the scaling with individual fragment size, which is prohibited by the use of RPA. Possible ways to improve this could include developing method-specific basis sets to minimize the problem, or translating the problem to a densityfunctional theory setting, where RPA correlation is routinely used. This would, however, remove the ability to decompose the excitations into different terms. In addition, the Cholesky decomposition required for the density fitting procedure becomes a bottleneck for very large systems. Again, this could be alleviated by utilising massive parallelization. In this way, it would become possible to routinely treat systems involving many hundreds of molecules in an accurate manner, and to gain physical insight into the main underlying physical effects through energy decomposition. 


\section{Acknowledgement}

The authors thank the Engineering and Physical Sciences Research Council (UK) for a postgraduate studentship awarded to R.A.S.

\section{Supporting Information Available}

Interaction energies and energy decompositions, both tabulated and in raw formats; errors and calibration graphs for local thresholds; geometries of the complexes in Figure 8; Cartesian coordinates of the CCSD/aVDZ optimized geometries for the S66 set.

\section{References}

(1) Full, R. J.; Autumn, K.; Liang, Y. A.; Hsieh, S. T.; Zesch, W.; Chan, W. P.; Kenny, T. W.; Fearing, R. Adhesive force of a single gecko foot-hair. Nature 2000, 405, 681-685.

(2) Autumn, K.; Sitti, M.; Liang, Y. A.; Peattie, A. M.; Hansen, W. R.; Sponberg, S.; Kenny, T. W.; Fearing, R.; Israelachvili, J. N.; Full, R. J. Evidence for van der Waals adhesion in gecko setae. Proc. Natl. Acad. Sci. U S A 2002, 99, 12252-12256.

(3) Rozitis, B.; MacLennan, E.; Emery, J. P. Cohesive forces prevent the rotational breakup of rubble-pile asteroid (29075) 1950 DA. Nature 2014, 512, 174-176.

(4) DiStasio, R. A.; von Lilienfeld, O. A.; Tkatchenko, A. Collective many-body van der Waals interactions in molecular systems. Proc. Natl. Acad. Sci. USA 2012, 109, 14791-14795.

(5) Muthukumar, M.; Ober, C.; Thomas, E. Competing Interactions and Levels of Ordering in Self-Organizing Polymeric Materials. Science 1997, 27\%, 1225-1232. 
(6) Stone, A. J. The Theory of Intermolecular Forces, 2nd ed.; OUP, 2013.

(7) Hohenstein, E. G.; Sherrill, C. D. Wavefunction methods for noncovalent interactions. Wiley Interdiscip. Rev. Comput. Mol. Sci. 2012, 2, 304-326.

(8) Yang, L.; Adam, C.; Nichol, G. S.; Cockroft, S. L. How much do van der Waals dispersion forces contribute to molecular recognition in solution? Nat. Chem. 2013, 5, 1006-1010.

(9) Jurečka, P.; Šponer, J.; Černý, J.; Hobza, P. Benchmark database of accurate (MP2 and $\operatorname{CCSD}(\mathrm{T})$ complete basis set limit) interaction energies of small model complexes, DNA base pairs, and amino acid pairs. Phys. Chem. Chem. Phys. 2006, 8, 1985-1993.

(10) Brauer, B.; Kesharwani, M. K.; Kozuch, S.; Martin, J. M. L. The S66x8 benchmark for noncovalent interactions revisited: explicitly correlated ab initio methods and density functional theory. Phys. Chem. Chem. Phys. 2016, 18, 20905-20925.

(11) Kesharwani, M. K.; Karton, A.; Sylvetsky, N.; Martin, J. M. L. The S66 Non-Covalent Interactions Benchmark Reconsidered Using Explicitly Correlated Methods Near the Basis Set Limit. Aust. J. Chem. 2018, 71, 238-248.

(12) Grimme, S. Density functional theory with London dispersion corrections. Wiley Interdiscip. Rev. Comput. Mol. Sci. 2011, 1, 211-228.

(13) Burns, L. A.; Mayagoitia, A. V.; Sumpter, B. G.; Sherrill, C. D. Density-functional approaches to noncovalent interactions: A comparison of dispersion corrections (DFTD), exchange-hole dipole moment (XDM) theory, and specialized functionals. J. Chem. Phys. 2011, 134, 084107.

(14) Riley, K. E.; Pitoňák, M.; Jurečka, P.; Hobza, P. Stabilization and Structure Calculations for Noncovalent Interactions in Extended Molecular Systems Based on Wave Function and Density Functional Theories. Chem. Rev. 2010, 110, 5023-5063. 
(15) Riley, K. E.; Hobza, P. Noncovalent interactions in biochemistry. Wiley Interdiscip. Rev. Comput. Mol. Sci. 2011, 1, 3-17.

(16) Grimme, S.; Hansen, A.; Brandenburg, J. G.; Bannwarth, C. Dispersion-Corrected Mean-Field Electronic Structure Methods. Chem. Rev. 2016, 116, 5105-5154.

(17) Granatier, J.; Pitoňák, M.; Hobza, P. Accuracy of Several Wave Function and Density Functional Theory Methods for Description of Noncovalent Interaction of Saturated and Unsaturated Hydrocarbon Dimers. J. Chem. Theory Comput. 2012, 8, 2282-2292.

(18) Hopkins, B. W.; Tschumper, G. S. Ab Initio Studies of $\pi \ldots \pi$ Interactions: The Effects of Quadruple Excitations. J. Phys. Chem. A 2004, 108, 2941-2948.

(19) Pitoňák, M.; Neogrády, P.; Řezáč, J.; Jurečka, P.; Urban, M.; Hobza, P. Benzene Dimer: High-Level Wave Function and Density Functional Theory Calculations. J. Chem. Theory Comput. 2008, 4, 1829-1834.

(20) de Jong, W. A.; Bylaska, E.; Govind, N.; Janssen, C. L.; Kowalski, K.; Miller, T.; Nielsen, I. M. B.; van Dam, H. J. J.; Veryazov, V.; Lindh, R. Utilizing high performance computing for chemistry: parallel computational chemistry. Phys. Chem. Chem. Phys. 2010, 12, 6896.

(21) Asadchev, A.; Gordon, M. S. Fast and Flexible Coupled Cluster Implementation. J. Chem. Theory Comput. 2013, 9, 3385-3392.

(22) Harding, M. E.; Metzroth, T.; Gauss, J.; Auer, A. A. Parallel Calculation of CCSD and CCSD(T) Analytic First and Second Derivatives. J. Chem. Theory Comput. 2008, 4, $64-74$.

(23) Eriksen, J. J.; Baudin, P.; Ettenhuber, P.; Kristensen, K.; Kjærgaard, T.; Jørgensen, P. Linear-Scaling Coupled Cluster with Perturbative Triple Excitations: The Divide- 
Expand-Consolidate CCSD(T) Model. J. Chem. Theory Comput. 2015, 11, 29842993.

(24) Schwilk, M.; Ma, Q.; Köppl, C.; Werner, H.-J. Scalable Electron Correlation Methods. 3. Efficient and Accurate Parallel Local Coupled Cluster with Pair Natural Orbitals (PNO-LCCSD). J. Chem. Theory Comput. 2017, 13, 3650-3675.

(25) Klopper, W.; Manby, F. R.; Ten-No, S.; Valeev, E. F. R12 methods in explicitly correlated molecular electronic structure theory. Int. Rev. Phys. Chem. 2006, 25, $427-468$.

(26) Bischoff, F. A.; Wolfsegger, S.; Tew, D. P.; Klopper, W. Assessment of basis sets for F12 explicitly-correlated molecular electronic-structure methods. Mol. Phys. 2009, 107, 963-975.

(27) Kong, L.; Bischoff, F. A.; Valeev, E. F. Explicitly correlated R12/F12 methods for electronic structure. Chem. Rev. 2012, 112, 75-107.

(28) Dunlap, B. I.; Connolly, J. W. D.; Sabin, J. R. On the applicability of LCAO-X methods to molecules containing transition metal atoms: The nickel atom and nickel hydride. Int. J. Quantum Chem. 1977, 12, 81-87.

(29) Dunlap, B. I.; Connolly, J. W. D.; Sabin, J. R. On some approximations in applications of X $\alpha$ theory. J. Chem. Phys. 1979, 71, 3396-3402.

(30) Weigend, F. A fully direct RI-HF algorithm: Implementation, optimised auxiliary basis sets, demonstration of accuracy and efficiency. Phys. Chem. Chem. Phys. 2002, 4, 4285-4291.

(31) Polly, R.; Werner, H.-J.; Manby, F. R.; Knowles, P. J. Fast Hartree-Fock theory using local density fitting approximations. Mol. Phys. 2004, 102, 2311-2321. 
(32) Köppl, C.; Werner, H.-J. Parallel and low-order scaling implementation of HartreeFock exchange using local density fitting. J. Chem. Theory Comput. 2016, 12, 31223134.

(33) Feyereisen, M.; Fitzgerald, G.; Komornicki, A. Use of approximate integrals in ab initio theory. An application in MP2 energy calculations. Chem. Phys. Lett. 1993, 208, 359-363.

(34) Werner, H.-J.; Manby, F. R.; Knowles, P. J. Fast linear scaling second-order MøllerPlesset perturbation theory (MP2) using local and density fitting approximations. J. Chem. Phys. 2003, 118, 8149-8160.

(35) Manby, F. R. Density fitting in second-order linear-r ${ }_{12}$ Møller-Plesset perturbation theory. J. Chem. Phys. 2003, 119, 4607-4613.

(36) Schutski, R.; Zhao, J.; Henderson, T. M.; Scuseria, G. E. Tensor-structured coupled cluster theory. J. Chem. Phys. 2017, 147, 184113.

(37) Pulay, P. Localizability of dynamic electron correlation. Chem. Phys. Lett. 1983, 100, $151-154$.

(38) Høyvik, I. M.; Jørgensen, P. Characterization and Generation of Local Occupied and Virtual Hartree-Fock Orbitals. Chem. Rev. 2016, 116, 3306-3327.

(39) Beran, G. J. O.; Hirata, S. Fragment and localized orbital methods in electronic structure theory. Phys. Chem. Chem. Phys. 2012, 14, 7559.

(40) Kjærgaard, T.; Baudin, P.; Bykov, D.; Kristensen, K.; Jørgensen, P. The divideexpand-consolidate coupled cluster scheme. Wiley Interdiscip. Rev. Comput. Mol. Sci. 2017, e1319.

(41) Schmitz, G.; Hättig, C.; Tew, D. P. Explicitly correlated PNO-MP2 and PNO-CCSD 
and their application to the S66 set and large molecular systems. Phys. Chem. Chem. Phys. 2014, 16, 22167-22178.

(42) Tew, D. P. Explicitly correlated coupled-cluster theory with Brueckner orbitals. J. Chem. Phys. 2016, 145, 074103.

(43) Schutz, M.; Lindh, R.; Werner, H.-J. Integral-direct electron correlation methods. Mol. Phys. 1999, 96, 719-733.

(44) Hättig, C.; Tew, D. P.; Helmich, B. Local explicitly correlated second- and third-order Møller-Plesset perturbation theory with pair natural orbitals. J. Chem. Phys. 2012, 136, 204105.

(45) Ouyang, J. F.; Bettens, R. P. A. When are Many-Body Effects Significant? J. Chem. Theory Comput. 2016, 12, 5860-5867.

(46) Ouyang, J. F.; Cvitkovic, M. W.; Bettens, R. P. A. Trouble with the many-body expansion. J. Chem. Theory Comput. 2014, 10, 3699-3707.

(47) Schütz, M.; Rauhut, G.; Werner, H.-J. Local Treatment of Electron Correlation in Molecular Clusters: Structures and Stabilities of $\left(\mathrm{H}_{2} \mathrm{O}\right)_{n}, n=2-4$. J. Phys. Chem. A 1998, 102, 5997-6003.

(48) Ouyang, J. F.; Bettens, R. P. A. Many-body basis set superposition effect. J. Chem. Theory Comput. 2015, 11, 5132-5143.

(49) Boys, S.; Bernardi, F. The calculation of small molecular interactions by the differences of separate total energies. Some procedures with reduced errors. Mol. Phys. 1970, 19, $553-566$.

(50) Mayer, I. The chemical Hamiltonian approach for treating the BSSE problem of intermolecular interactions. Int. J. Quantum Chem. 1998, 70, 41-63. 
(51) Jeziorski, B.; Moszynski, R.; Szalewicz, K. Perturbation Theory Approach to Intermolecular Potential Energy Surfaces of van der Waals Complexes. Chem. Rev. 1994, 94, 1187-1930.

(52) Hesselmann, A.; Jansen, G.; Schütz, M. Density-functional theory-symmetry-adapted intermolecular perturbation theory with density fitting: A new efficient method to study intermolecular interaction energies. J. Chem. Phys. 2005, 122, 014103.

(53) Williams, H. L.; Chabalowski, C. F. Using Kohn-Sham Orbitals in Symmetry-Adapted Perturbation Theory to Investigate Intermolecular Interactions. J. Phys. Chem. A 2001, 105, 646-659.

(54) Podeszwa, R.; Szalewicz, K. Accurate interaction energies for argon, krypton, and benzene dimers from perturbation theory based on the Kohn-Sham model. Chem. Phys. Lett. 2005, 412, 488-493.

(55) Mo, Y.; Song, L.; Lin, Y. Block-localized wavefunction (BLW) method at the density functional theory (DFT) level. J. Phys. Chem. A 2007, 111, 8291-8301.

(56) Sironi, M.; Genoni, A.; Civera, M.; Pieraccini, S.; Ghitti, M. Extremely localized molecular orbitals: Theory and applications. Theor. Chem. Acc. 2007, 117, 685-698.

(57) Meyer, B.; Guillot, B.; Ruiz-Lopez, M. F.; Genoni, A. Libraries of Extremely Localized Molecular Orbitals. 1. Model Molecules Approximation and Molecular Orbitals Transferability. J. Chem. Theory Comput. 2016, 12, 1052-1067.

(58) Khaliullin, R. Z.; Head-Gordon, M.; Bell, A. T. An efficient self-consistent field method for large systems of weakly interacting components. J. Chem. Phys. 2006, 124, 204105.

(59) Saebo, S.; Pulay, P. Local Treatment of Electron Correlation. Ann. Rev. Phys. Chem. 1993, 44, 213-236. 
(60) Ettenhuber, P.; Baudin, P.; Kjærgaard, T.; Jørgensen, P.; Kristensen, K. Orbital spaces in the divide-expand-consolidate coupled cluster method. J. Chem. Phys. 2016, $144,164116$.

(61) Wolinski, K.; Pulay, P. Second-order Møller-Plesset calculations with dual basis sets. J. Chem. Phys. 2003, 118, 9497-9503.

(62) Steele, R. P.; Shao, Y.; DiStasio, R. A.; Head-Gordon, M. Dual-basis analytic gradients. 1. Self-consistent field theory. J. Phys. Chem. A 2006, 110, 13915-13922.

(63) Deng, J.; Gill, P. M. W. A new approach to dual-basis second-order Møller-Plesset calculations. J. Chem. Phys. 2011, 134, 081103.

(64) Hamza, A.; Vibók, Á.; Halász, G. J.; Mayer, I. BSSE-free SCF theories: A comment. J. Mol. Struct. THEOCHEM 2000, 501-502, 427-434.

(65) Mayer, I. Hermitian "chemical" Hamiltonian: an alternative ab initio method. Theor. Chem. Acc. 2015, $134,86$.

(66) Hohenstein, E. G.; Sherrill, C. D. Density fitting and Cholesky decomposition approximations in symmetry-adapted perturbation theory: Implementation and application to probe the nature of $\pi-\pi$ interactions in linear acenes. J. Chem. Phys. 2010, 132, 184111.

(67) Mayer, I.; Vibök, Á. SCF theory of intermolecular interactions without basis set superposition error. Chem. Phys. Lett. 1987, 136, 115-121.

(68) Stoll, H.; Wagenblast, G.; Preuss, H. On the Use of Local Basis-Sets for Localized Molecular-Orbitals. Theor. Chim. Acta. 1980, 57, 169-178.

(69) Cullen, J. M. An examination of the effects of basis set and charge transfer in hydrogenbonded dimers with a constrained Hartree-Fock method. Int. J. Quantum Chem. 1991, 40, 193-207. 
(70) Gianinetti, E.; Raimondi, M.; Tornaghi, E. Modification of the Roothaan equations to exclude BSSE from molecular interaction calculations. Int. J. Quantum Chem. 1996, $60,157-166$.

(71) Famulari, A.; Gianinetti, E.; Raimondi, M.; Sironi, M. Implementation of gradientoptimization algorithms and force constant computations in BSSE-free direct and conventional SCF approaches. Int. J. Quantum Chem. 1998, 69, 151-158.

(72) Nagata, T.; Takahashi, O.; Saito, K.; Iwata, S. Basis set superposition error free self-consistent field method for molecular interaction in multi-component systems: Projection operator formalism. J. Chem. Phys. 2001, 115, 3553-3560.

(73) Nagata, T.; Iwata, S. Perturbation expansion theory corrected from basis set superposition error. I. Locally projected excited orbitals and single excitations. J. Chem. Phys. 2004, 120, 3555-3562.

(74) Iwata, S. The single excitation perturbation expansion theory based on the locally projected molecular orbitals for molecular interaction: Comparison with the counterpoise corrected energy. Chem. Phys. Lett. 2006, 431, 204-209.

(75) Iwata, S. Absolutely local excited orbitals in the higher order perturbation expansion for the molecular interaction. J. Phys. Chem. B 2008, 112, 16104-16109.

(76) Iwata, S. Absolutely local occupied and excited molecular orbitals in the third-order single excitation perturbation theory for molecular interaction. J. Phys. Chem. A 2010, 114, 8697-8704.

(77) Khaliullin, R. Z.; Bell, A. T.; Head-Gordon, M. Analysis of charge transfer effects in molecular complexes based on absolutely localized molecular orbitals. J. Chem. Phys. 2008, 128, 184112 . 
(78) Khaliullin, R. Z.; Cobar, E. A.; Lochan, R. C.; Bell, A. T.; Head-Gordon, M. Unravelling the origin of intermolecular interactions using absolutely localized molecular orbitals. J. Phys. Chem. A 2007, 111, 8753-8765.

(79) Azar, R. J.; Head-Gordon, M. An energy decomposition analysis for intermolecular interactions from an absolutely localized molecular orbital reference at the coupledcluster singles and doubles level. J. Chem. Phys. 2012, 136, 024103.

(80) Thirman, J.; Head-Gordon, M. Efficient Implementation of Energy Decomposition Analysis for Second-Order Møller-Plesset Perturbation Theory and Application to Anion- $\pi$ Interactions. J. Phys. Chem. A 2017, 121, 717-728.

(81) Thirman, J.; Head-Gordon, M. An energy decomposition analysis for second-order Møller-Plesset perturbation theory based on absolutely localized molecular orbitals. J. Chem. Phys. 2015, 143, 084124.

(82) Thirman, J.; Engelage, E.; Huber, S. M.; Head-Gordon, M. Characterizing the interplay of Pauli repulsion, electrostatics, dispersion and charge transfer in halogen bonding with energy decomposition analysis. Phys. Chem. Chem. Phys. 2018, 20, 905-915.

(83) Mao, Y.; Ge, Q.; Horn, P. R.; Head-Gordon, M. On the computational characterization of charge-transfer effects in noncovalently bound molecular complexes. J. Chem. Theory Comput. 2018, 14, 2401-2417.

(84) Horn, P. R.; Sundstrom, E. J.; Baker, T. A.; Head-Gordon, M. Unrestricted absolutely localized molecular orbitals for energy decomposition analysis: Theory and applications to intermolecular interactions involving radicals. J. Chem. Phys. 2013, 138, 134119.

(85) Mulliken, R. S. Electronic Population Analysis on LCAO-MO Molecular Wave Functions. I. J. Chem. Phys. 1955, 23, 1833-1840. 
(86) Löwdin, P.-O. On the Non-Orthogonality Problem Connected with the Use of Atomic Wave Functions in the Theory of Molecules and Crystals. J. Chem. Phys. 1950, 18, $365-375$.

(87) Havriliak, S.; King, H. F. Rydberg radicals. 1. Frozen-core model for Rydberg levels of the ammonium radical. J. Am. Chem. Soc. 1983, 105, 4-12.

(88) Steele, R. P.; Head-Gordon, M.; Tully, J. C. Ab Initio Molecular Dynamics with Dual Basis Set Methods. J. Phys. Chem. A 2010, 114, 11853-11860.

(89) Zhang, J.; Dolg, M. Third-Order Incremental Dual-Basis Set Zero-Buffer Approach: An Accurate and Efficient Way To Obtain CCSD and CCSD(T) Energies. J. Chem. Theory Comput. 2013, 9, 2992-3003.

(90) Deng, J.; Gilbert, A. T. B.; Gill, P. M. W. MP2[V]: A Simple Approximation to Second-Order Møller-Plesset Perturbation Theory. J. Chem. Theory Comput. 2015, 11, 1639-1644.

(91) Liang, W.; Head-Gordon, M. An exact reformulation of the diagonalization step in electronic structure calculations as a set of second order nonlinear equations. J. Chem. Phys. 2004, 120, 10379-10384.

(92) Hermann, J.; DiStasio, R. A.; Tkatchenko, A. First-Principles Models for van der Waals Interactions in Molecules and Materials: Concepts, Theory, and Applications. Chem. Rev. 2017, 117, 4714-4758.

(93) McLachlan, A. D.; Ball, M. A. Time-Dependent Hartree-Fock Theory for Molecules. Rev. Mod. Phys. 1964, 36, 844-855.

(94) Toulouse, J.; Gerber, I. C.; Jansen, G.; Savin, A.; Ángyán, J. G. Adiabatic-Connection Fluctuation-Dissipation Density-Functional Theory Based on Range Separation. Phys. Rev. Lett. 2009, 102, 096404. 
(95) Bohm, D.; Pines, D. A Collective Description of Electron Interactions: III. Coulomb Interactions in a Degenerate Electron Gas. Phys. Rev. 1953, 92, 609-625.

(96) Bohm, D.; Pines, D. A Collective Description of Electron Interactions. I. Magnetic Interactions. Phys. Rev. 1951, 82, 625-634.

(97) Pines, D.; Bohm, D. A Collective Description of Electron Interactions: II. Collective vs. Individual Particle Aspects of the Interactions. Phys. Rev. 1952, 85, 338-353.

(98) Pines, D. A Collective Description of Electron Interactions: IV. Electron Interaction in Metals. Phys. Rev. 1953, 92, 626-636.

(99) Tkatchenko, A.; Ambrosetti, A.; DiStasio, R. A. Interatomic methods for the dispersion energy derived from the adiabatic connection fluctuation-dissipation theorem. J. Chem. Phys. 2013, 138, 074106.

(100) Ambrosetti, A.; Reilly, A. M.; DiStasio, R. A.; Tkatchenko, A. Long-range correlation energy calculated from coupled atomic response functions. J. Chem. Phys. 2014, 140, $18 \mathrm{~A} 508$.

(101) Tkatchenko, A.; DiStasio, R. A.; Car, R.; Scheffler, M. Accurate and Efficient Method for Many-Body van der Waals Interactions. Phys. Rev. Lett. 2012, 108, 236402.

(102) Toulouse, J.; Zhu, W.; Ángyán, J. G.; Savin, A. Range-separated density-functional theory with the random-phase approximation: Detailed formalism and illustrative applications. Phys. Rev. A 2010, 82, 032502.

(103) Ruiz, V. G.; Liu, W.; Tkatchenko, A. Density-functional theory with screened van der Waals interactions applied to atomic and molecular adsorbates on close-packed and non-close-packed surfaces. Phys. Rev. B 2016, 93, 035118.

(104) Chen, G. P.; Voora, V. K.; Agee, M. M.; Balasubramani, S. G.; Furche, F. RandomPhase Approximation Methods. Ann. Rev. Phys. Chem. 2017, 68, 421-445. 
(105) Eshuis, H.; Bates, J. E.; Furche, F. Electron correlation methods based on the random phase approximation. Theor. Chem. Acc. 2012, 131, 1084.

(106) Hehn, A.-S.; Tew, D. P.; Klopper, W. Explicitly correlated ring-coupled-clusterdoubles theory. J. Chem. Phys. 2015, 142, 194106.

(107) Jansen, G.; Liu, R.-F.; Ángyán, J. G. On the equivalence of ring-coupled cluster and adiabatic connection fluctuation-dissipation theorem random phase approximation correlation energy expressions. J. Chem. Phys. 2010, 133, 154106.

(108) Scuseria, G. E.; Henderson, T. M.; Sorensen, D. C. The ground state correlation energy of the random phase approximation from a ring coupled cluster doubles approach. $J$. Chem. Phys. 2008, 129, 231101.

(109) Scuseria, G. E.; Henderson, T. M.; Bulik, I. W. Particle-particle and quasiparticle random phase approximations: Connections to coupled cluster theory. J. Chem. Phys. 2013, 139, 104113.

(110) Toulouse, J.; Zhu, W.; Savin, A.; Jansen, G.; Ángyán, J. G. Closed-shell ring coupled cluster doubles theory with range separation applied on weak intermolecular interactions. J. Chem. Phys. 2011, 135, 084119.

(111) Langreth, D.; Perdew, J. The exchange-correlation energy of a metallic surface. Solid State Commun. 1975, 17, 1425-1429.

(112) Høyvik, I.-M.; Olsen, J.; Jørgensen, P. Generalising localisation schemes of orthogonal orbitals to the localisation of non-orthogonal orbitals. Mol. Phys. 2017, 115, 16-25.

(113) Jódar, L. A formula for the general solution of Riccati type matrix differential equations. Systems $\&$ Control Letters 1989, 12, 39-43. 
(114) Ren, X.; Rinke, P.; Joas, C.; Scheffler, M. Random-phase approximation and its applications in computational chemistry and materials science. J. Mater. Sci. 2012, 47, $7447-7471$.

(115) Singwi, K. S.; Tosi, M. P.; Land, R. H.; Sjolander, A. Electron Correlations at Metallic Densities. Phys. Rev. 1968, 176, 589-599.

(116) Ángyán, J. G.; Liu, R.-F.; Toulouse, J.; Jansen, G. Correlation Energy Expressions from the Adiabatic-Connection Fluctuation-Dissipation Theorem Approach. J. Chem. Theory Comput. 2011, 7, 3116-3130.

(117) Paier, J.; Janesko, B. G.; Henderson, T. M.; Scuseria, G. E.; Gruneis, A.; Kresse, G. Hybrid functionals including random phase approximation correlation and secondorder screened exchange. J. Chem. Phys. 2010, 132, 094103.

(118) Helgaker, T.; Jorgensen, P.; Olsen, J. Molecular Electronic Structure Theory; John Wiley \& Sons, Inc.: Chichester, UK, 2000.

(119) Golub, G.; van Loan, C. Matrix Computations, 4th ed.; John Hopkins University Press: Baltimore, USA, 2012.

(120) Shaw, R. A. GAMMA, an electronic structure code for intermolecular interactions. https://github.com/robashaw/gamma, date accessed: 22 May 2019.

(121) Valeev, E. F. Libint: A library for the evaluation of molecular integrals of manybody operators over Gaussian functions. http://libint.valeyev.net/, version 2.3.1, date accessed: 22 May 2019.

(122) Guennebaud, G.; Jacob, B.; Others, Eigen v3. http://eigen.tuxfamily.org, date accessed: 22 May 2019. 
(123) Hampel, C.; Peterson, K. A.; Werner, H.-J. A comparison of the efficiency and accuracy of the quadratic configuration interaction (QCISD), coupled cluster (CCSD), and Brueckner coupled cluster (BCCD) methods. Chem. Phys. Lett. 1992, 190, 1-12.

(124) Deegan, M. J.; Knowles, P. J. Perturbative corrections to account for triple excitations in closed and open shell coupled cluster theories. Chem. Phys. Lett. 1994, 227, 321326.

(125) Zhao, Y.; Truhlar, D. G. The M06 suite of density functionals for main group thermochemistry, thermochemical kinetics, noncovalent interactions, excited states, and transition elements: two new functionals and systematic testing of four M06-class functionals and 12 other function. Theor. Chem. Acc. 2008, 120, 215-241.

(126) Werner, H.-J.; Knowles, P. J.; Knizia, G.; Manby, F. R.; Schütz, M.; Celani, P.; Györffy, W.; Kats, D.; Korona, T.; Lindh, R.; Mitrushenkov, A.; Rauhut, G.; Shamasundar, K. R.; Adler, T. B.; Amos, R. D.; Bernhardsson, A.; Berning, A.; Cooper, D. L.; Deegan, M. J. O.; Dobbyn, A. J.; Eckert, F.; Goll, E.; Hampel, C.; Hesselmann, A.; Hetzer, G.; Hrenar, T.; Jansen, G.; Köppl, C.; Liu, Y.; Lloyd, A. W.; Mata, R. A.; May, A. J.; McNicholas, S. J.; Meyer, W.; Mura, M. E.; Nicklass, A.; O’Neill, D. P.; Palmieri, P.; Peng, D.; Pflüger, K.; Pitzer, R.; Reiher, M.; Shiozaki, T.; Stoll, H.; Stone, A. J.; Tarroni, R.; Thorsteinsson, T.; Wang, M. MOLPRO, version 2015.1, a package of ab initio programs. 2015.

(127) Hohenstein, E. G.; Parrish, R. M.; Sherrill, C. D.; Turney, J. M.; Schaefer, H. F. Large-scale symmetry-adapted perturbation theory computations via density fitting and Laplace transformation techniques: Investigating the fundamental forces of DNAintercalator interactions. J. Chem. Phys. 2011, 135, 174107.

(128) Parrish, R. M.; Burns, L. A.; Smith, D. G. A.; Simmonett, A. C.; DePrince, A. E.; Hohenstein, E. G.; Bozkaya, U.; Sokolov, A. Y.; Di Remigio, R.; Richard, R. M.; 
Gonthier, J. F.; James, A. M.; McAlexander, H. R.; Kumar, A.; Saitow, M.; Wang, X.; Pritchard, B. P.; Verma, P.; Schaefer, H. F.; Patkowski, K.; King, R. A.; Valeev, E. F.; Evangelista, F. A.; Turney, J. M.; Crawford, T. D.; Sherrill, C. D. Psi4 1.1: An OpenSource Electronic Structure Program Emphasizing Automation, Advanced Libraries, and Interoperability. J. Chem. Theory Comput. 2017, 13, 3185-3197.

(129) Woon, D. E.; Dunning, T. H. Gaussian basis sets for use in correlated molecular calculations. III. The atoms aluminum through argon. J. Chem. Phys. 1993, 98, 13581371.

(130) Dunning, T. H. Gaussian basis sets for use in correlated molecular calculations. I. The atoms boron through neon and hydrogen. J. Chem. Phys. 1989, 90, 1007-1023.

(131) Dunning, T. H.; Peterson, K. A.; Wilson, A. K. Gaussian basis sets for use in correlated molecular calculations. X. The atoms aluminum through argon revisited. J. Chem. Phys. 2001, 114, 9244-9253.

(132) Weigend, F. Accurate Coulomb-fitting basis sets for H to Rn. Phys. Chem. Chem. Phys. 2006, 8, 1057.

(133) Weigend, F. Hartree-Fock exchange fitting basis sets for H to Rn. J. Comput. Chem. 2008, 29, 167-175.

(134) Weigend, F.; Köhn, A.; Hättig, C. Efficient use of the correlation consistent basis sets in resolution of the identity MP2 calculations. J. Chem. Phys. 2002, 116, 3175-3183.

(135) Řezáč, J.; Riley, K. E.; Hobza, P. S66: A Well-balanced Database of Benchmark Interaction Energies Relevant to Biomolecular Structures. J. Chem. Theory Comput. 2011, 7, 2427-2438.

(136) Manna, D.; Kesharwani, M. K.; Sylvetsky, N.; Martin, J. M. L. Conventional and 
Explicitly Correlated ab Initio Benchmark Study on Water Clusters: Revision of the BEGDB and WATER27 Data Sets. J. Chem. Theory Comput. 2017, 13, 3136-3152.

(137) Tew, D. P. Quasi-robust local density fitting. J. Chem. Phys. 2018, 148, 011102.

(138) Shaw, R. A.; Hill, J. G.; Legon, A. C. Halogen Bonding with Phosphine: Evidence for Mulliken Inner Complexes and the Importance of Relaxation Energy. J. Phys. Chem. A 2016, 120, 8461-8468.

(139) Stone, A. J.; Misquitta, A. J. Charge-transfer in Symmetry-Adapted Perturbation Theory. Chem. Phys. Lett. 2009, 473, 201-205. 


\title{
Supporting information for:
}

\section{A linear-scaling method for non-covalent interactions: An efficient combination of}

\section{absolutely localized molecular orbitals and a local random phase approximation approach}

\author{
Robert A. Shaw* and J. Grant Hill* \\ Department of Chemistry, University of Sheffield, Sheffield S3 7HF, U.K. \\ E-mail: rashaw1@sheffield.ac.uk; grant.hill@sheffield.ac.uk
}

\section{Contents}

1 Additional files

2 Interaction energies

3 Energy decompositions

4 Local thresholds, errors, and domain sizes

5 Geometries 


\section{Additional files}

The supporting information for this paper also includes cartesian coordinates of the CCSD/aVDZ geometries for the S66 set of complexes. These can be found in xyzfiles.tar.gz, and are labelled as DonorAcceptor.xyz. In addition to this, all interaction energies and energy decompositions can be found in CSV format in the datasets folder. 


\section{Interaction energies}

The following shorthands are used for molecule names to save space in tables: benzene, Bz.; uracil, Ur.; peptide, Pep.; pyridine, Pyr.; pentane, Pent.

Table S1: Uncorrelated interaction energies in $\mathrm{kcal} \mathrm{mol}^{-1}$ for the molecules in the S66 database using aVDZ basis sets. Counterpoise-corrected Hartree-Fock (HF) is compared with the ALMO + charge transfer (CT) energies, along with the components: CT and local exchange correction (XCorrect) energies, and the ALMO energy (including XCorrect).

\begin{tabular}{|c|c|c|c|c|c|c|c|}
\hline ID & Donor & Acceptor & $\mathrm{HF}$ & $\mathrm{ALMO}+\mathrm{CT}$ & ALMO & XCorrect & $\mathrm{CT}$ \\
\hline 1 & $\mathrm{H}_{2} \mathrm{O}$ & $\mathrm{H}_{2} \mathrm{O}$ & -3.7319 & -3.7622 & -2.8451 & 0.0734 & -0.9171 \\
\hline 2 & $\mathrm{H}_{2} \mathrm{O}$ & $\mathrm{MeOH}$ & -3.7816 & -3.8694 & -2.6902 & 0.0176 & -1.1792 \\
\hline 3 & $\mathrm{H}_{2} \mathrm{O}$ & $\mathrm{MeNH}_{2}$ & -4.7275 & -4.7039 & -2.9612 & 0.0533 & -1.7427 \\
\hline 4 & $\mathrm{H}_{2} \mathrm{O}$ & Pep. & -5.9168 & -6.0111 & -4.3691 & 0.4926 & -1.6419 \\
\hline 5 & $\mathrm{MeOH}$ & $\mathrm{MeOH}$ & -3.5277 & -3.6347 & -2.4209 & 0.0132 & -1.2139 \\
\hline 6 & $\mathrm{MeOH}$ & $\mathrm{MeNH}_{2}$ & -4.2225 & -4.2921 & -2.4212 & 0.0019 & -1.8709 \\
\hline 7 & $\mathrm{MeOH}$ & Pep. & -5.0263 & -5.1416 & -3.4414 & -1.0950 & -1.7002 \\
\hline 8 & $\mathrm{MeOH}$ & $\mathrm{H}_{2} \mathrm{O}$ & -3.5005 & -3.5491 & -2.6023 & 0.0364 & -0.9468 \\
\hline 9 & $\mathrm{MeNH}_{2}$ & $\mathrm{MeOH}$ & -0.8049 & -1.0769 & -0.3308 & 0.0088 & -0.7461 \\
\hline 10 & $\mathrm{MeNH}_{2}$ & $\mathrm{MeNH}_{2}$ & -1.0839 & -1.3463 & -0.3690 & 0.0107 & -0.9773 \\
\hline 11 & $\mathrm{MeNH}_{2}$ & Pep. & -1.2614 & -1.6540 & -0.5340 & 0.0188 & -1.1201 \\
\hline 12 & $\mathrm{MeNH}_{2}$ & $\mathrm{H}_{2} \mathrm{O}$ & -4.7543 & -4.7765 & -2.9145 & 0.0069 & -1.8620 \\
\hline 13 & Pep. & $\mathrm{MeOH}$ & -3.0324 & -3.3530 & -2.2052 & -0.7091 & -1.1478 \\
\hline 14 & Pep. & $\mathrm{MeNH}_{2}$ & -3.4517 & -3.7538 & -2.0242 & -0.8402 & -1.7296 \\
\hline 15 & Pep. & Pep. & -4.4891 & -4.9058 & -3.2124 & -0.9758 & -1.6934 \\
\hline 16 & Pep. & $\mathrm{H}_{2} \mathrm{O}$ & -3.3832 & -3.6300 & -2.7369 & -0.5422 & -0.8931 \\
\hline 17 & Ur. & Ur. BP & -13.3455 & -13.1239 & -8.4967 & -1.8010 & -4.6272 \\
\hline 18 & $\mathrm{H}_{2} \mathrm{O}$ & Pyr. & -4.4513 & -4.4266 & -2.8885 & 0.0552 & -1.5382 \\
\hline 19 & $\mathrm{MeOH}$ & Pyr. & -4.1911 & -4.1797 & -2.5478 & -0.9639 & -1.6320 \\
\hline
\end{tabular}




\begin{tabular}{|c|c|c|c|c|c|c|c|}
\hline ID & Donor & Acceptor & $\mathrm{HF}$ & $\mathrm{ALMO}+\mathrm{CT}$ & ALMO & XCorrect & $\mathrm{CT}$ \\
\hline 20 & $\mathrm{AcOH}$ & $\mathrm{AcOH}$ & -15.1633 & -14.5807 & -9.6235 & -2.1712 & -4.9572 \\
\hline 21 & $\mathrm{AcNH}_{2}$ & $\mathrm{AcNH}_{2}$ & -12.6204 & -12.4206 & -9.1219 & -1.6554 & -3.2987 \\
\hline 22 & $\mathrm{AcOH}$ & Ur. & -15.6672 & -15.3103 & -10.8245 & -1.8963 & -4.4858 \\
\hline 23 & $\mathrm{AcNH}_{2}$ & Ur. & -15.5342 & -15.1060 & -11.1846 & -2.0708 & -3.9214 \\
\hline 24 & $\mathrm{Bz}$. & Bz. pipi & 4.3623 & 3.2432 & 4.6232 & -0.5409 & -1.3800 \\
\hline 25 & Pyr. & Pyr. pipi & 3.6080 & 2.7399 & 4.0081 & 0.1186 & -1.2682 \\
\hline 26 & Ur. & Ur. pipi & 3.1525 & 1.6438 & 4.0957 & 0.1092 & -2.4518 \\
\hline 27 & Bz. & Pyr. pipi & 3.8047 & 2.8045 & 4.1153 & -0.5102 & -1.3107 \\
\hline 28 & $\mathrm{Bz}$. & Ur. pipi & 7.4911 & 5.9739 & 8.2716 & 0.4336 & -2.2978 \\
\hline 29 & Pyr. & Ur. pipi & 5.7661 & 4.5512 & 6.7267 & 0.2956 & -2.1755 \\
\hline 30 & Bz. & Ethene & 3.3546 & 3.0932 & 3.7338 & 0.0107 & -0.6407 \\
\hline 31 & Ur. & Ethene & 1.4819 & 1.1805 & 1.7861 & 0.0420 & -0.6056 \\
\hline 32 & Ur. & Ethyne & 0.1296 & -0.0970 & 0.4225 & 0.0358 & -0.5195 \\
\hline 33 & Pyr. & Ethene & 5.6291 & 5.2505 & 6.4783 & 0.0176 & -1.2278 \\
\hline 34 & Pent. & Pent. & 4.8194 & 4.0807 & 5.2571 & 0.2041 & -1.1764 \\
\hline 35 & Neopent. & Pent. & 3.7167 & 3.0561 & 4.0945 & -0.6181 & -1.0384 \\
\hline 36 & Neopent. & Neopent. & 2.8743 & 2.3238 & 3.2047 & 0.5158 & -0.8808 \\
\hline 37 & Cyclopent. & Neopent. & 3.7731 & 3.1339 & 4.1566 & -0.5974 & -1.0227 \\
\hline 38 & Cyclopent. & Cyclopent. & 3.7608 & 3.2100 & 4.1813 & -0.6752 & -0.9713 \\
\hline 39 & $\mathrm{Bz}$. & Cyclopent. & 3.5157 & 2.4057 & 3.7659 & 0.3552 & -1.3601 \\
\hline 40 & $\mathrm{Bz}$. & Neopent. & 2.9327 & 1.8818 & 3.1615 & -0.6338 & -1.2797 \\
\hline 41 & Ur. & Pent. & 5.6986 & 4.5772 & 6.3512 & 0.4436 & -1.7741 \\
\hline 42 & Ur. & Cyclopent. & 5.3810 & 4.3184 & 5.9176 & 1.0235 & -1.5992 \\
\hline 43 & Ur. & Neopent. & 3.7471 & 2.8434 & 4.1778 & -0.7769 & -1.3343 \\
\hline 44 & Ethene & Pent. & 1.9421 & 1.6493 & 2.1535 & 0.0075 & -0.5043 \\
\hline
\end{tabular}




\begin{tabular}{|c|c|c|c|c|c|c|c|}
\hline ID & Donor & Acceptor & $\mathrm{HF}$ & $\mathrm{ALMO}+\mathrm{CT}$ & ALMO & XCorrect & $\mathrm{CT}$ \\
\hline 45 & Ethyne & Pent. & 1.4908 & 1.2487 & 1.6786 & 0.0126 & -0.4299 \\
\hline 46 & Pep. & Pent. & 4.2688 & 3.3366 & 4.8002 & 0.3031 & -1.4635 \\
\hline 47 & $\mathrm{Bz}$. & Bz. TS & 1.8333 & 1.1770 & 2.0213 & -0.2629 & -0.8443 \\
\hline 48 & Pyr. & Pyr. TS & 1.0617 & 0.4361 & 1.3900 & -0.5045 & -0.9539 \\
\hline 49 & $\mathrm{Bz}$ & Pyr. TS & 1.3050 & 0.3301 & 1.4576 & -0.4876 & -1.1275 \\
\hline 50 & $\mathrm{Bz}$. & Ethyne CH-pi & -0.0664 & -0.9465 & 0.0886 & -0.4405 & -1.0351 \\
\hline 51 & Ethyne & Ethyne TS & -0.4296 & -0.6047 & -0.1271 & -0.2792 & -0.4776 \\
\hline 52 & $\mathrm{Bz}$ & AcOHOH-pi & -0.5764 & -1.4018 & -0.2156 & -0.4826 & -1.1862 \\
\hline 53 & $\mathrm{Bz}$. & $\mathrm{AcNH}_{2} \mathrm{NH}-\mathrm{pi}$ & 0.0772 & -0.5431 & 0.3249 & -0.7599 & -0.8680 \\
\hline 54 & $\mathrm{Bz}$. & $\mathrm{H}_{2} \mathrm{OOH}-\mathrm{pi}$ & -0.8199 & -1.3626 & -0.6412 & 0.3753 & -0.7214 \\
\hline 55 & $\mathrm{Bz}$. & MeOHOH-pi & 0.3270 & -0.2328 & 0.6622 & 0.3985 & -0.8950 \\
\hline 56 & $\mathrm{Bz}$. & $\mathrm{MeNH}_{2} \mathrm{NH}-\mathrm{pi}$ & 1.3754 & 0.5799 & 1.5495 & 0.0458 & -0.9695 \\
\hline 57 & $\mathrm{Bz}$. & Pep. NH-pi & 3.9026 & 2.5914 & 4.4824 & 0.0075 & -1.8909 \\
\hline 58 & Pyr. & Pyr. CH-N & -1.2536 & -1.5936 & -0.7315 & -0.5773 & -0.8622 \\
\hline 59 & Ethyne & $\mathrm{H}_{2} \mathrm{OCH}-\mathrm{O}$ & -2.3106 & -2.5380 & -1.8731 & -0.3338 & -0.6649 \\
\hline 60 & Ethyne & AcOHOH-pi & -2.8891 & -2.9724 & -1.9249 & -0.8340 & -1.0475 \\
\hline 61 & Pent. & $\mathrm{AcOH}$ & 2.3075 & 1.5962 & 2.5982 & 0.0157 & -1.0021 \\
\hline 62 & Pent. & $\mathrm{AcNH}_{2}$ & 2.2796 & 1.7092 & 2.6923 & 0.1349 & -0.9830 \\
\hline 63 & $\mathrm{Bz}$. & $\mathrm{AcOH}$ & 1.7734 & 0.7621 & 1.9988 & -0.4826 & -1.2367 \\
\hline 64 & Pep. & Ethene & 0.5280 & 0.2065 & 0.8991 & 0.0107 & -0.6927 \\
\hline 65 & Pyr. & Ethyne & -2.7363 & -2.9583 & -1.9821 & -0.4179 & -0.9762 \\
\hline 66 & $\mathrm{MeNH}_{2}$ & Pyr. & 0.9678 & 0.4815 & 1.4558 & 0.0151 & -0.9743 \\
\hline
\end{tabular}




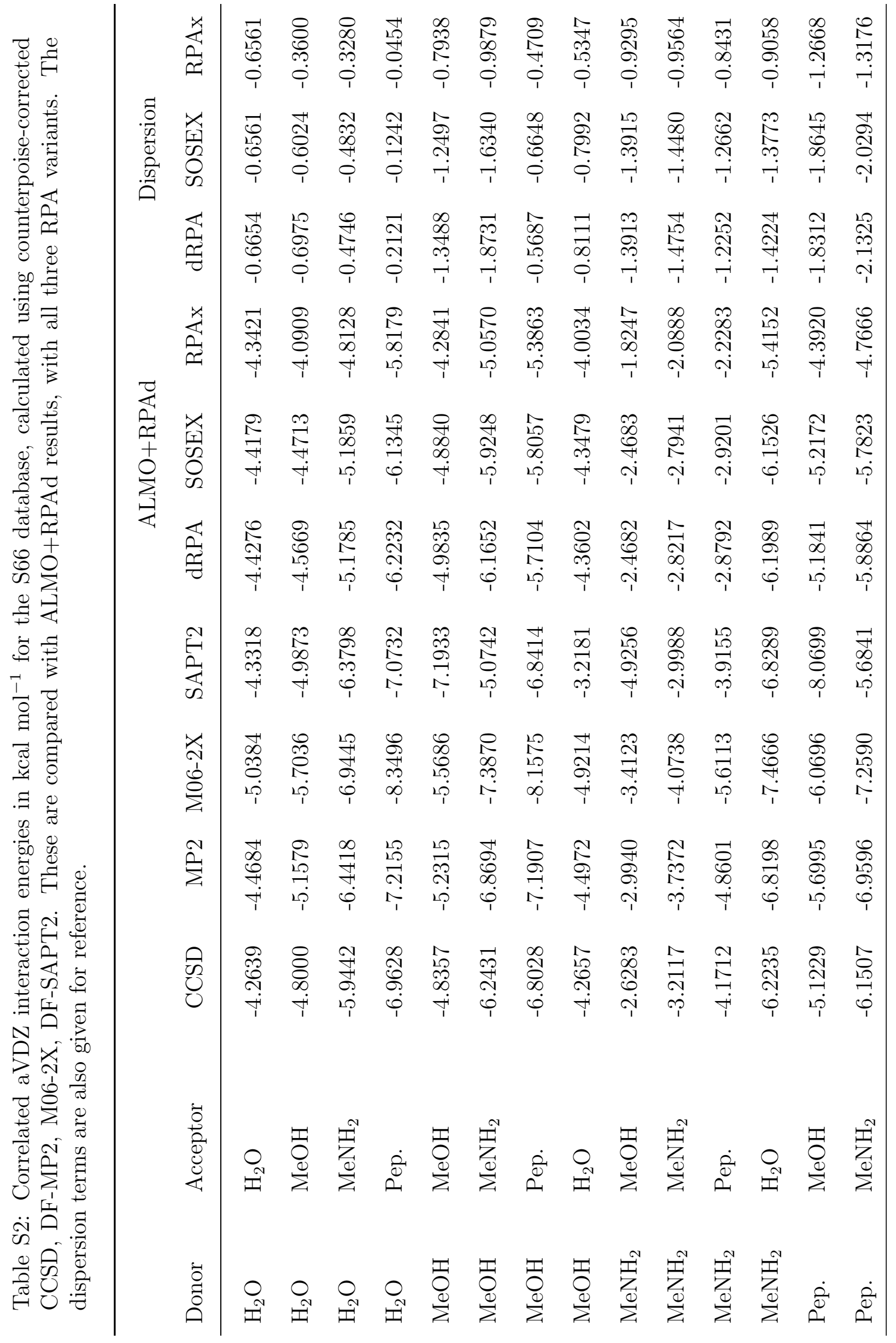




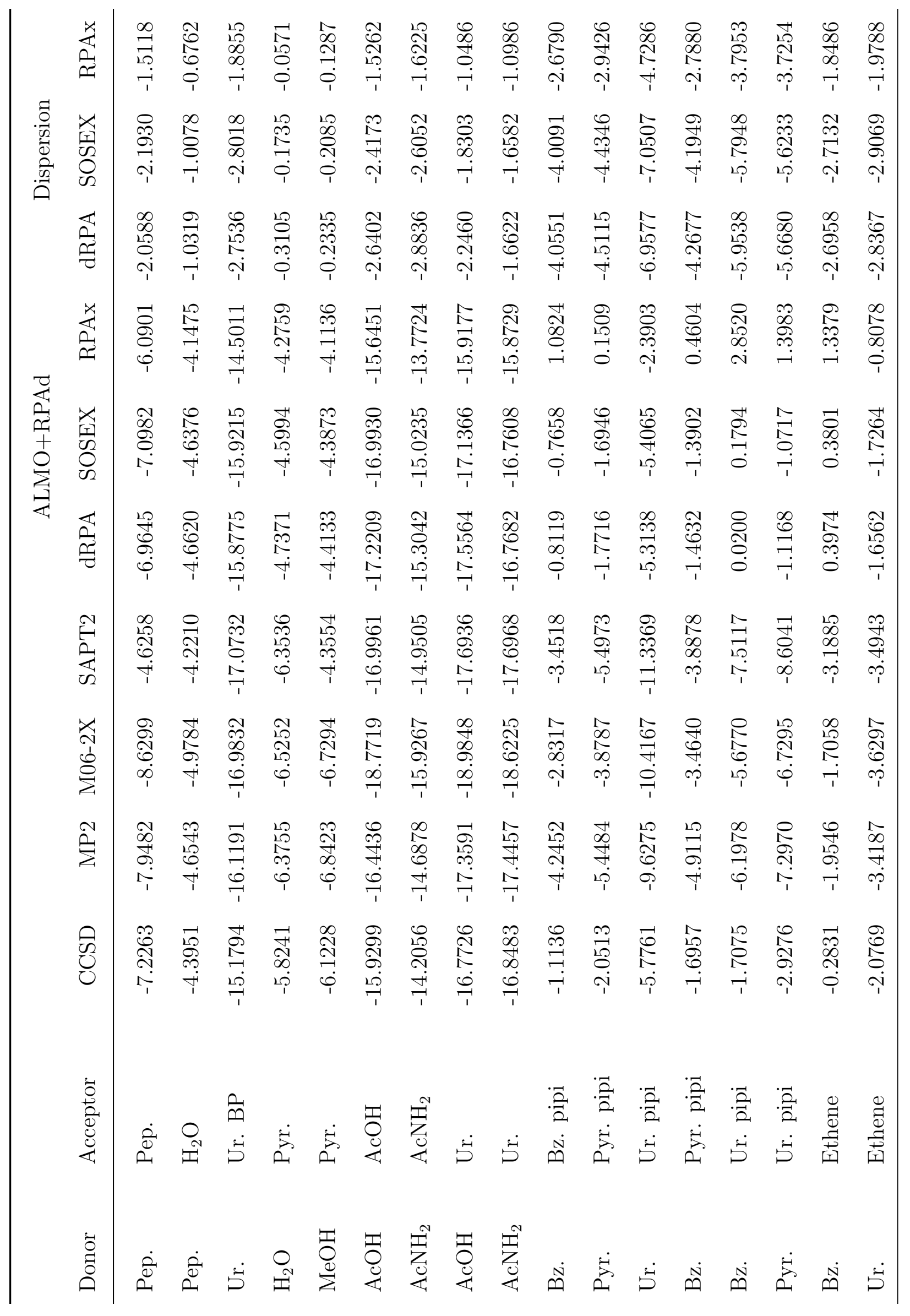




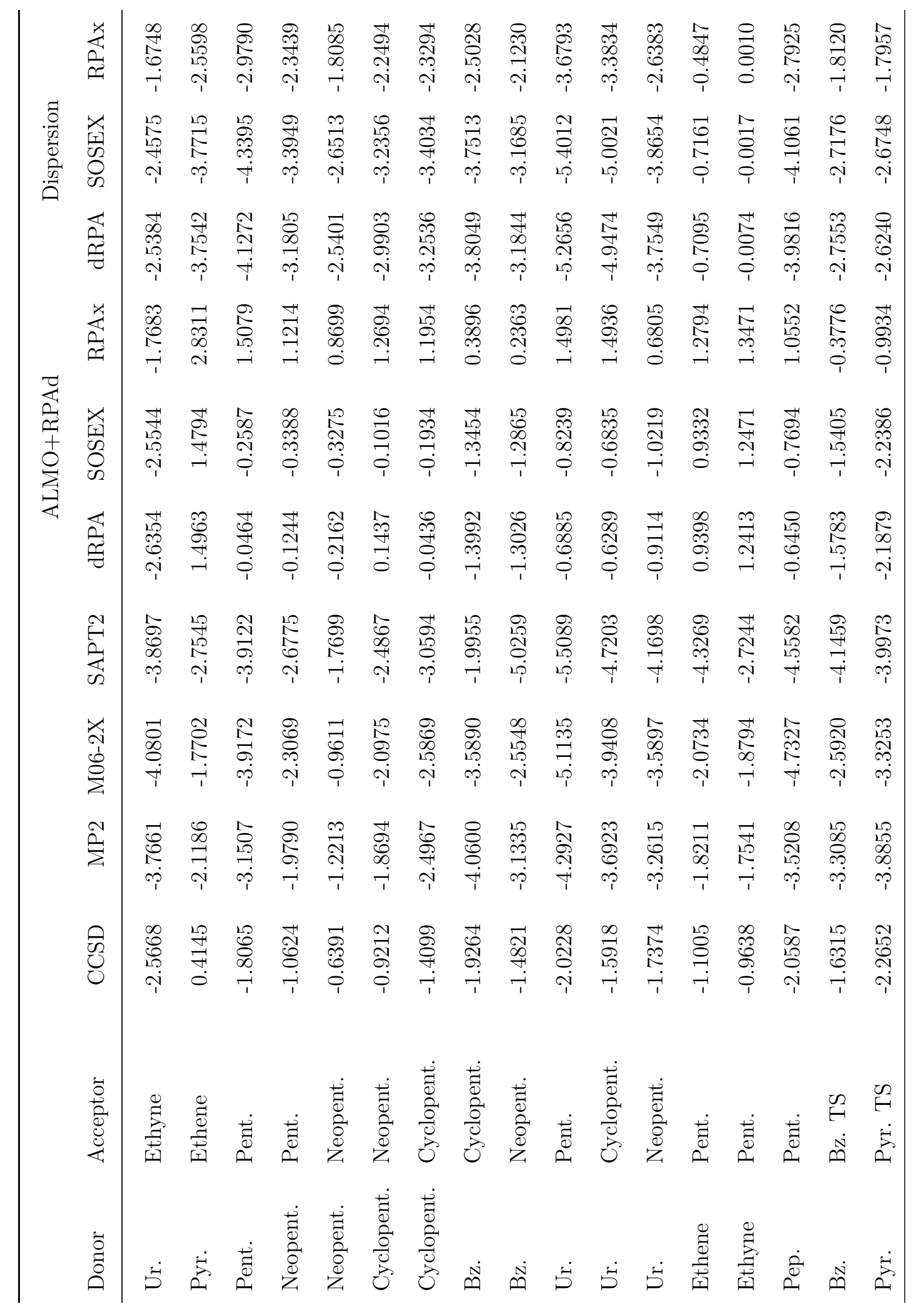




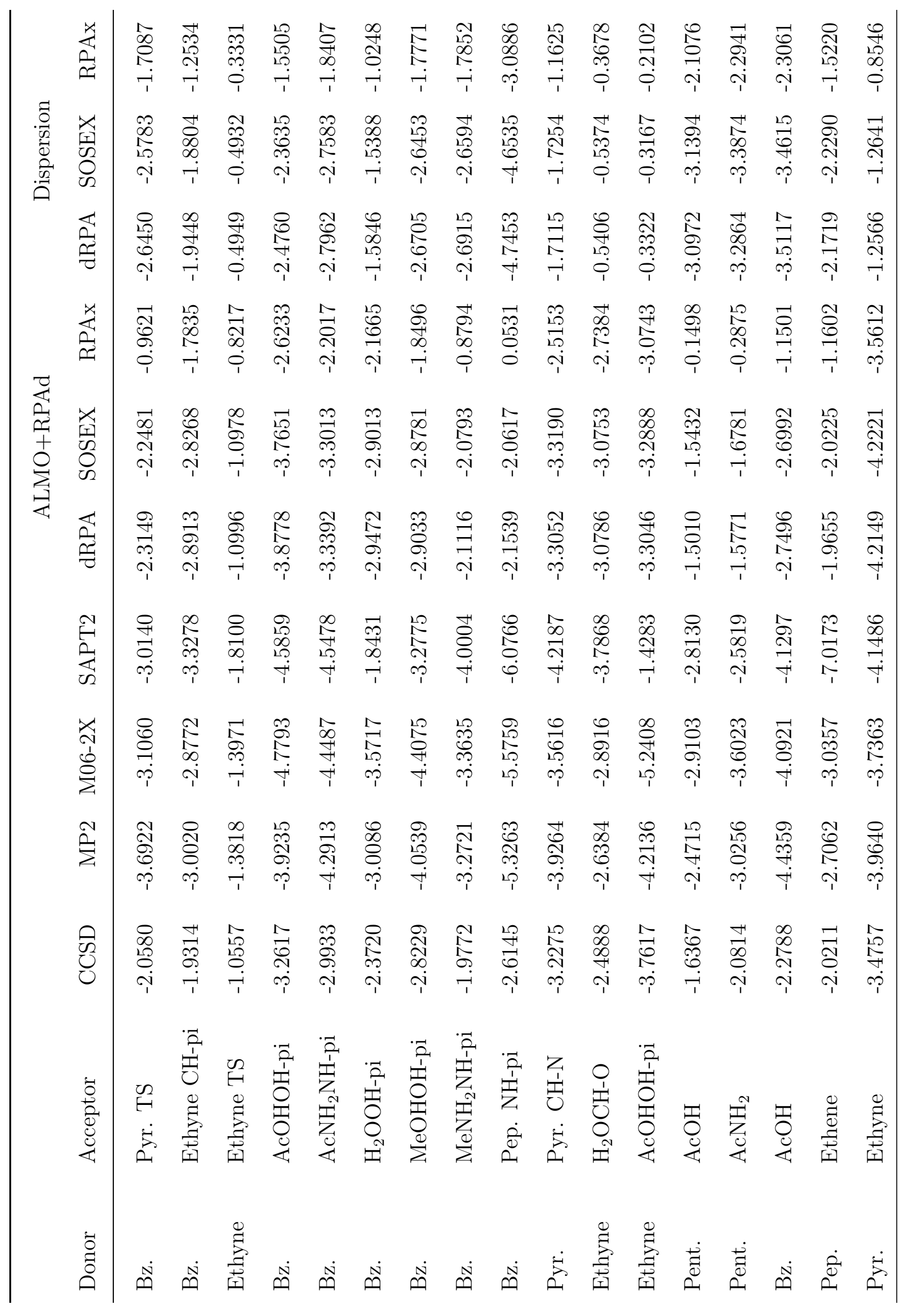




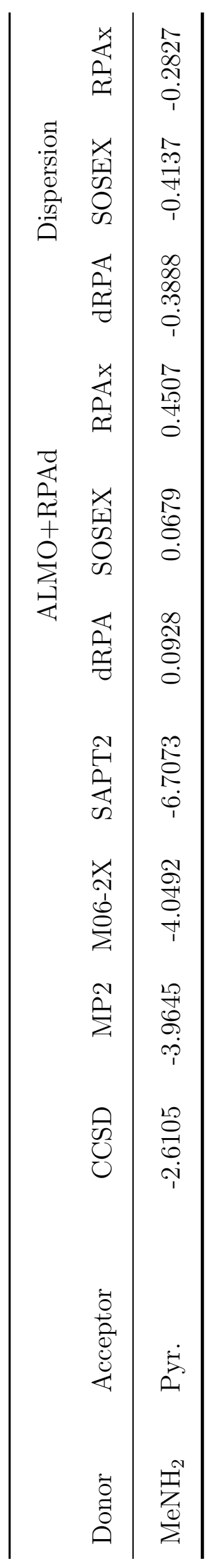


Table S3: Correlated aVDZ interaction energies in $\mathrm{kcal} \mathrm{mol}^{-1}$ for the S66 database, calculated using c CCSD, DF-MP2, and M06-2X, with and without the counterpoise correction.

\begin{tabular}{|c|c|c|c|c|c|c|c|}
\hline \multirow[b]{2}{*}{ Donor } & \multirow[b]{2}{*}{ Acceptor } & \multicolumn{2}{|c|}{ CCSD } & \multicolumn{2}{|c|}{ MP2 } & \multicolumn{2}{|c|}{ M06-2X } \\
\hline & & $\mathrm{CP}$ & No CP & $\mathrm{CP}$ & No CP & $\mathrm{CP}$ & No CP \\
\hline $\mathrm{H}_{2} \mathrm{O}$ & $\mathrm{H}_{2} \mathrm{O}$ & -4.2639 & -4.2652 & -4.4684 & -4.2652 & -5.0384 & -5.0387 \\
\hline $\mathrm{H}_{2} \mathrm{O}$ & $\mathrm{MeOH}$ & -4.8000 & -4.8016 & -5.1579 & -4.8016 & -5.7036 & -5.7042 \\
\hline $\mathrm{H}_{2} \mathrm{O}$ & $\mathrm{MeNH}_{2}$ & -5.9442 & -5.9459 & -6.4418 & -5.9459 & -6.9445 & -6.9451 \\
\hline $\mathrm{H}_{2} \mathrm{O}$ & Pep. & -6.9628 & -6.9649 & -7.2155 & -6.9649 & -8.3496 & -8.3504 \\
\hline $\mathrm{MeOH}$ & $\mathrm{MeOH}$ & -4.8357 & -4.8376 & -5.2315 & -4.8376 & -5.5686 & -5.5692 \\
\hline $\mathrm{MeOH}$ & $\mathrm{MeNH}_{2}$ & -6.2431 & -6.2455 & -6.8694 & -6.2455 & -7.3870 & -7.3880 \\
\hline $\mathrm{MeOH}$ & Pep. & -6.8028 & -6.8055 & -7.1907 & -6.8055 & -8.1575 & -8.1583 \\
\hline $\mathrm{MeOH}$ & $\mathrm{H}_{2} \mathrm{O}$ & -4.2657 & -4.2670 & -4.4972 & -4.2670 & -4.9214 & -4.9217 \\
\hline $\mathrm{MeNH}_{2}$ & $\mathrm{MeOH}$ & -2.6283 & -2.6302 & -2.9940 & -2.6302 & -3.4123 & -3.4131 \\
\hline $\mathrm{MeNH}_{2}$ & $\mathrm{MeNH}_{2}$ & -3.2117 & -3.2139 & -3.7372 & -3.2139 & -4.0738 & -4.0747 \\
\hline $\mathrm{MeNH}_{2}$ & Pep. & -4.1712 & -4.1744 & -4.8601 & -4.1744 & -5.6113 & -5.6125 \\
\hline $\mathrm{MeNH}_{2}$ & $\mathrm{H}_{2} \mathrm{O}$ & -6.2235 & -6.2255 & -6.8198 & -6.2255 & -7.4666 & -7.4674 \\
\hline Pep. & $\mathrm{MeOH}$ & -5.1229 & -5.1256 & -5.6995 & -5.1256 & -6.0696 & -6.0707 \\
\hline Pep. & $\mathrm{MeNH}_{2}$ & -6.1507 & -6.1540 & -6.9596 & -6.1540 & -7.2590 & -7.2604 \\
\hline Pep. & Pep. & -7.2263 & -7.2304 & -7.9482 & -7.2304 & -8.6299 & -8.6313 \\
\hline Pep. & $\mathrm{H}_{2} \mathrm{O}$ & -4.3951 & -4.3971 & -4.6543 & -4.3971 & -4.9784 & -4.9792 \\
\hline Ur. & Ur. BP & -15.1794 & -15.1845 & -16.1191 & -15.1845 & -16.9832 & -16.9894 \\
\hline $\mathrm{H}_{2} \mathrm{O}$ & Pyr. & -5.8241 & -5.8260 & -6.3755 & -5.8260 & -6.5252 & -6.5258 \\
\hline $\mathrm{MeOH}$ & Pyr. & -6.1228 & -6.1250 & -6.8423 & -6.1250 & -6.7294 & -6.7301 \\
\hline $\mathrm{AcOH}$ & $\mathrm{AcOH}$ & -15.9299 & -15.9338 & -16.4436 & -15.9338 & -18.7719 & -18.7730 \\
\hline $\mathrm{AcNH}_{2}$ & $\mathrm{AcNH}_{2}$ & -14.2056 & -14.2090 & -14.6878 & -14.2090 & -15.9267 & -15.9279 \\
\hline $\mathrm{AcOH}$ & Ur. & -16.7726 & -16.7769 & -17.3591 & -16.7769 & -18.9848 & -18.9860 \\
\hline
\end{tabular}




\begin{tabular}{|c|c|c|c|c|c|c|c|}
\hline \multirow[b]{2}{*}{ Donor } & \multirow[b]{2}{*}{ Acceptor } & \multicolumn{2}{|c|}{ CCSD } & \multicolumn{2}{|c|}{ MP2 } & \multicolumn{2}{|c|}{ M06-2X } \\
\hline & & $\mathrm{CP}$ & No CP & $\mathrm{CP}$ & No CP & $\mathrm{CP}$ & No CP \\
\hline $\mathrm{AcNH}_{2}$ & Ur. & -16.8483 & -16.8524 & -17.4457 & -16.8524 & -18.6225 & -18.6239 \\
\hline $\mathrm{Bz}$. & Bz. pipi & -1.1136 & -1.1189 & -4.2452 & -1.1189 & -2.8317 & -2.8333 \\
\hline Pyr. & Pyr. pipi & -2.0513 & -2.0564 & -5.4484 & -2.0564 & -3.8787 & -3.8803 \\
\hline Ur. & Ur. pipi & -5.7761 & -5.7817 & -9.6275 & -5.7817 & -10.4167 & -10.4256 \\
\hline Bz. & Pyr. pipi & -1.6957 & -1.7009 & -4.9115 & -1.7009 & -3.4640 & -3.4655 \\
\hline $\mathrm{Bz}$. & Ur. pipi & -1.7075 & -1.7171 & -6.1978 & -1.7171 & -5.6770 & -5.6841 \\
\hline Pyr. & Ur. pipi & -2.9276 & -2.9350 & -7.2970 & -2.9350 & -6.7295 & -6.7334 \\
\hline $\mathrm{Bz}$. & Ethene & -0.2831 & -0.2857 & -1.9546 & -0.2857 & -1.7058 & -1.7067 \\
\hline Ur. & Ethene & -2.0769 & -2.0805 & -3.4187 & -2.0805 & -3.6297 & -3.6308 \\
\hline Ur. & Ethyne & -2.5668 & -2.5697 & -3.7661 & -2.5697 & -4.0801 & -4.0808 \\
\hline Pyr. & Ethene & 0.4145 & 0.4079 & -2.1186 & 0.4079 & -1.7702 & -1.7776 \\
\hline Pent. & Pent. & -1.8065 & -1.8088 & -3.1507 & -1.8088 & -3.9172 & -3.9197 \\
\hline Neopent. & Pent. & -1.0624 & -1.0657 & -1.9790 & -1.0657 & -2.3069 & -2.3156 \\
\hline Neopent. & Neopent. & -0.6391 & -0.6460 & -1.2213 & -0.6460 & -0.9611 & -0.9615 \\
\hline Cyclopent. & Neopent. & -0.9212 & -0.9232 & -1.8694 & -0.9232 & -2.0975 & -2.1017 \\
\hline Cyclopent. & Cyclopent. & -1.4099 & -1.4113 & -2.4967 & -1.4113 & -2.5869 & -2.5880 \\
\hline Bz. & Cyclopent. & -1.9264 & -1.9324 & -4.0600 & -1.9324 & -3.5890 & -3.5909 \\
\hline $\mathrm{Bz}$. & Neopent. & -1.4821 & -1.4876 & -3.1335 & -1.4876 & -2.5548 & -2.5566 \\
\hline Ur. & Pent. & -2.0228 & -2.0263 & -4.2927 & -2.0263 & -5.1135 & -5.1213 \\
\hline Ur. & Cyclopent. & -1.5918 & -1.5929 & -3.6923 & -1.5929 & -3.9408 & -3.9479 \\
\hline Ur. & Neopent. & -1.7374 & -1.7377 & -3.2615 & -1.7377 & -3.5897 & -3.5912 \\
\hline Ethene & Pent. & -1.1005 & -1.1030 & -1.8211 & -1.1030 & -2.0734 & -2.0744 \\
\hline Ethyne & Pent. & -0.9638 & -0.9659 & -1.7541 & -0.9659 & -1.8794 & -1.8800 \\
\hline Pep. & Pent. & -2.0587 & -2.0666 & -3.5208 & -2.0666 & -4.7327 & -4.7367 \\
\hline
\end{tabular}




\begin{tabular}{|c|c|c|c|c|c|c|c|}
\hline \multirow[b]{2}{*}{ Donor } & \multirow[b]{2}{*}{ Acceptor } & \multicolumn{2}{|c|}{ CCSD } & \multicolumn{2}{|c|}{ MP2 } & \multicolumn{2}{|c|}{ M06-2X } \\
\hline & & $\mathrm{CP}$ & No CP & $\mathrm{CP}$ & No CP & $\mathrm{CP}$ & No CP \\
\hline Bz. & Bz. TS & -1.6315 & -1.6366 & -3.3085 & -1.6366 & -2.5920 & -2.5934 \\
\hline Pyr. & Pyr. TS & -2.2652 & -2.2696 & -3.8855 & -2.2696 & -3.3253 & -3.3266 \\
\hline $\mathrm{Bz}$. & Pyr. TS & -2.0580 & -2.0632 & -3.6922 & -2.0632 & -3.1060 & -3.1075 \\
\hline $\mathrm{Bz}$. & Ethyne CH-pi & -1.9314 & -1.9356 & -3.0020 & -1.9356 & -2.8772 & -2.8784 \\
\hline Ethyne & Ethyne TS & -1.0557 & -1.0572 & -1.3818 & -1.0572 & -1.3971 & -1.3976 \\
\hline $\mathrm{Bz}$. & AcOHOH-pi & -3.2617 & -3.2660 & -3.9235 & -3.2660 & -4.7793 & -4.7801 \\
\hline Bz. & $\mathrm{AcNH}_{2} \mathrm{NH}-\mathrm{pi}$ & -2.9933 & -2.9978 & -4.2913 & -2.9978 & -4.4487 & -4.4500 \\
\hline Bz. & $\mathrm{H}_{2} \mathrm{OOH}-\mathrm{pi}$ & -2.3720 & -2.3747 & -3.0086 & -2.3747 & -3.5717 & -3.5723 \\
\hline $\mathrm{Bz}$. & MeOHOH-pi & -2.8229 & -2.8270 & -4.0539 & -2.8270 & -4.4075 & -4.4086 \\
\hline $\mathrm{Bz}$. & $\mathrm{MeNH}_{2} \mathrm{NH}-\mathrm{pi}$ & -1.9772 & -1.9812 & -3.2721 & -1.9812 & -3.3635 & -3.3646 \\
\hline Bz. & Pep. NH-pi & -2.6145 & -2.6204 & -5.3263 & -2.6204 & -5.5759 & -5.5767 \\
\hline Pyr. & Pyr. CH-N & -3.2275 & -3.2304 & -3.9264 & -3.2304 & -3.5616 & -3.5627 \\
\hline Ethyne & $\mathrm{H}_{2} \mathrm{OCH}-\mathrm{O}$ & -2.4888 & -2.4902 & -2.6384 & -2.4902 & -2.8916 & -2.8923 \\
\hline Ethyne & AcOHOH-pi & -3.7617 & -3.7637 & -4.2136 & -3.7637 & -5.2408 & -5.2413 \\
\hline Pent. & $\mathrm{AcOH}$ & -1.6367 & -1.6410 & -2.4715 & -1.6410 & -2.9103 & -2.9120 \\
\hline Pent. & $\mathrm{AcNH}_{2}$ & -2.0814 & -2.0856 & -3.0256 & -2.0856 & -3.6023 & -3.6039 \\
\hline Bz. & $\mathrm{AcOH}$ & -2.2788 & -2.2842 & -4.4359 & -2.2842 & -4.0921 & -4.0938 \\
\hline Pep. & Ethene & -2.0211 & -2.0237 & -2.7062 & -2.0237 & -3.0357 & -3.0366 \\
\hline Pyr. & Ethyne & -3.4757 & -3.4778 & -3.9640 & -3.4778 & -3.7363 & -3.7374 \\
\hline $\mathrm{MeNH}_{2}$ & Pyr. & -2.6105 & -2.6139 & -3.9645 & -2.6139 & -4.0492 & -4.0502 \\
\hline
\end{tabular}




\section{$3 \quad$ Energy decompositions}

Table S4: The ratio of dispersion to polarization, $r_{\mathrm{D} / \mathrm{P}}$ for the complexes in the S66 database, calculated at the aVTZ level using DF-SAPT2 and ALMO+RPAxd. These are used to classify the systems as one of: electrostatically dominate $\left(\mathrm{E}, r_{\mathrm{D} / \mathrm{P}}<2\right)$; dispersive $(\mathrm{D}$, $\left.r_{\mathrm{D} / \mathrm{P}} \geq 8\right)$; or mixed $\left(\mathrm{M}, 2 \leq r_{\mathrm{D} / \mathrm{P}}<8\right)$. The four disagreeing cases are highlighted in blue. Note the SAPT polarization term is calculated as $E_{\mathrm{Ind} .}-E_{\mathrm{CT}}$.

\begin{tabular}{|c|c|c|c|c|c|}
\hline \multirow[b]{2}{*}{ Donor } & \multirow[b]{2}{*}{ Acceptor } & \multicolumn{2}{|c|}{$r_{\mathrm{D} / \mathrm{P}}$} & \multicolumn{2}{|c|}{ Classification } \\
\hline & & SAPT & ALMO & SAPT & ALMO \\
\hline $\mathrm{H}_{2} \mathrm{O}$ & $\mathrm{H}_{2} \mathrm{O}$ & 1.0996 & 1.1363 & $\mathrm{E}$ & $\mathrm{E}$ \\
\hline $\mathrm{H}_{2} \mathrm{O}$ & $\mathrm{MeOH}$ & 1.1895 & 0.7290 & $\mathrm{E}$ & $\mathrm{E}$ \\
\hline $\mathrm{H}_{2} \mathrm{O}$ & $\mathrm{MeNH}_{2}$ & 0.9225 & 0.5320 & $\mathrm{E}$ & $\mathrm{E}$ \\
\hline $\mathrm{H}_{2} \mathrm{O}$ & Pep. & 1.1286 & 0.0652 & $\mathrm{E}$ & $\mathrm{E}$ \\
\hline $\mathrm{MeOH}$ & $\mathrm{MeOH}$ & 1.2861 & 1.2525 & $\mathrm{E}$ & $\mathrm{E}$ \\
\hline $\mathrm{MeOH}$ & $\mathrm{MeNH}_{2}$ & 1.1180 & 1.0529 & $\mathrm{E}$ & $\mathrm{E}$ \\
\hline $\mathrm{MeOH}$ & Pep. & 1.2441 & 0.5944 & $\mathrm{E}$ & $\mathrm{E}$ \\
\hline $\mathrm{MeOH}$ & $\mathrm{H}_{2} \mathrm{O}$ & 1.1889 & 1.2113 & $\mathrm{E}$ & $\mathrm{E}$ \\
\hline $\mathrm{MeNH}_{2}$ & $\mathrm{MeOH}$ & 2.9022 & 2.9504 & M & M \\
\hline $\mathrm{MeNH}_{2}$ & $\mathrm{MeNH}_{2}$ & 2.7331 & 2.7504 & M & M \\
\hline $\mathrm{MeNH}_{2}$ & Pep. & 3.0486 & 1.6564 & M & $\mathrm{E}$ \\
\hline $\mathrm{MeNH}_{2}$ & $\mathrm{H}_{2} \mathrm{O}$ & 1.0336 & 1.0656 & $\mathrm{E}$ & $\mathrm{E}$ \\
\hline Pep. & $\mathrm{MeOH}$ & 1.9626 & 1.9777 & $\mathrm{E}$ & $\mathrm{E}$ \\
\hline Pep. & $\mathrm{MeNH}_{2}$ & 1.5598 & 1.5885 & $\mathrm{E}$ & $\mathrm{E}$ \\
\hline Pep. & Pep. & 1.7348 & 1.3800 & $\mathrm{E}$ & $\mathrm{E}$ \\
\hline Pep. & $\mathrm{H}_{2} \mathrm{O}$ & 1.6348 & 1.6477 & $\mathrm{E}$ & $\mathrm{E}$ \\
\hline Ur. & Ur. BP & 0.7991 & 0.6272 & $\mathrm{E}$ & $\mathrm{E}$ \\
\hline $\mathrm{H}_{2} \mathrm{O}$ & Pyr. & 1.0165 & 0.0375 & $\mathrm{E}$ & $\mathrm{E}$ \\
\hline $\mathrm{MeOH}$ & Pyr. & 1.1486 & 0.3009 & $\mathrm{E}$ & $\mathrm{E}$ \\
\hline
\end{tabular}




\begin{tabular}{|c|c|c|c|c|c|}
\hline \multirow[b]{2}{*}{ Donor } & \multirow[b]{2}{*}{ Acceptor } & \multicolumn{2}{|c|}{$r_{\mathrm{D} / \mathrm{P}}$} & \multicolumn{2}{|c|}{ Classification } \\
\hline & & SAPT & ALMO & SAPT & ALMO \\
\hline $\mathrm{AcOH}$ & $\mathrm{AcOH}$ & 0.5899 & 0.4789 & $\mathrm{E}$ & $\mathrm{E}$ \\
\hline $\mathrm{AcNH}_{2}$ & $\mathrm{AcNH}_{2}$ & 0.7992 & 0.6795 & $\mathrm{E}$ & $\mathrm{E}$ \\
\hline $\mathrm{AcOH}$ & Ur. & 0.6553 & 0.3490 & $\mathrm{E}$ & $\mathrm{E}$ \\
\hline $\mathrm{AcNH}_{2}$ & Ur. & 0.7291 & 0.3304 & $\mathrm{E}$ & $\mathrm{E}$ \\
\hline $\mathrm{Bz}$. & Bz. pipi & 14.2983 & 14.0566 & $\mathrm{D}$ & $\mathrm{D}$ \\
\hline Pyr. & Pyr. pipi & 12.6527 & 14.2959 & $\mathrm{D}$ & $\mathrm{D}$ \\
\hline Ur. & Ur. pipi & 7.8886 & 6.6198 & $\mathrm{M}$ & M \\
\hline Bz. & Pyr. pipi & 13.2797 & 14.1122 & $\mathrm{D}$ & $\mathrm{D}$ \\
\hline $\mathrm{Bz}$. & Ur. pipi & 10.9757 & 9.3313 & $\mathrm{D}$ & $\mathrm{D}$ \\
\hline Pyr. & Ur. pipi & 10.2527 & 8.7565 & $\mathrm{D}$ & $\mathrm{D}$ \\
\hline Bz. & Ethene & 12.2746 & 15.7291 & $\mathrm{D}$ & $\mathrm{D}$ \\
\hline Ur. & Ethene & 11.6850 & 12.4449 & $\mathrm{D}$ & $\mathrm{D}$ \\
\hline Ur. & Ethyne & 10.4475 & 9.7863 & $\mathrm{D}$ & $\mathrm{D}$ \\
\hline Pyr. & Ethene & 11.9918 & 17.8708 & $\mathrm{D}$ & $\mathrm{D}$ \\
\hline Pent. & Pent. & 20.9825 & 40.7263 & $\mathrm{D}$ & $\mathrm{D}$ \\
\hline Neopent. & Pent. & 18.8088 & 35.1521 & $\mathrm{D}$ & $\mathrm{D}$ \\
\hline Neopent. & Neopent. & 18.1156 & 35.6381 & $\mathrm{D}$ & $\mathrm{D}$ \\
\hline Cyclopent. & Neopent. & 18.0419 & 32.4876 & $\mathrm{D}$ & $\mathrm{D}$ \\
\hline Cyclopent. & Cyclopent. & 18.9463 & 39.0147 & $\mathrm{D}$ & $\mathrm{D}$ \\
\hline $\mathrm{Bz}$. & Cyclopent. & 14.1383 & 16.6807 & $\mathrm{D}$ & $\mathrm{D}$ \\
\hline $\mathrm{Bz}$. & Neopent. & 13.2838 & 15.9362 & $\mathrm{D}$ & $\mathrm{D}$ \\
\hline Ur. & Pent. & 12.3377 & 12.4501 & $\mathrm{D}$ & $\mathrm{D}$ \\
\hline Ur. & Cyclopent. & 14.9510 & 16.5343 & $\mathrm{D}$ & $\mathrm{D}$ \\
\hline Ur. & Neopent. & 14.3679 & 16.0482 & $\mathrm{D}$ & $\mathrm{D}$ \\
\hline
\end{tabular}




\begin{tabular}{|c|c|c|c|c|c|}
\hline \multirow[b]{2}{*}{ Donor } & \multirow[b]{2}{*}{ Acceptor } & \multicolumn{2}{|c|}{$r_{\mathrm{D} / \mathrm{P}}$} & \multicolumn{2}{|c|}{ Classification } \\
\hline & & SAPT & ALMO & SAPT & ALMO \\
\hline Ethene & Pent. & 14.7407 & 10.4116 & $\mathrm{D}$ & $\mathrm{D}$ \\
\hline Ethyne & Pent. & 12.8984 & 10.8721 & $\mathrm{D}$ & $\mathrm{D}$ \\
\hline Pep. & Pent. & 9.3676 & 8.9429 & $\mathrm{D}$ & $\mathrm{D}$ \\
\hline Bz. & Bz. TS & 10.1721 & 10.8393 & $\mathrm{D}$ & $\mathrm{D}$ \\
\hline Pyr. & Pyr. TS & 8.2129 & 8.1578 & $\mathrm{D}$ & $\mathrm{D}$ \\
\hline Bz. & Pyr. TS & 8.0469 & 7.8367 & $\mathrm{D}$ & M \\
\hline Bz. & Ethyne CH-pi & 4.5897 & 4.5801 & M & M \\
\hline Ethyne & Ethyne TS & 3.0189 & 3.4605 & M & M \\
\hline Bz. & AcOHOH-pi & 2.8023 & 2.8262 & M & M \\
\hline Bz. & $\mathrm{AcNH}_{2} \mathrm{NH}-\mathrm{pi}$ & 3.1488 & 2.9239 & M & M \\
\hline Bz. & $\mathrm{H}_{2} \mathrm{OOH}-\mathrm{pi}$ & 3.2660 & 3.5820 & M & M \\
\hline Bz. & MeOHOH-pi & 4.4019 & 4.7884 & M & M \\
\hline Bz. & $\mathrm{MeNH}_{2} \mathrm{NH}-\mathrm{pi}$ & 7.9365 & 9.1545 & M & $\mathrm{D}$ \\
\hline Bz. & Pep. NH-pi & 5.3659 & 5.0200 & M & M \\
\hline Pyr. & Pyr. CH-N & 3.1774 & 2.7165 & M & M \\
\hline Ethyne & $\mathrm{H}_{2} \mathrm{OCH}-\mathrm{O}$ & 1.6967 & 1.8127 & $\mathrm{E}$ & $\mathrm{E}$ \\
\hline Ethyne & AcOHOH-pi & 1.6756 & 0.5967 & $\mathrm{E}$ & $\mathrm{E}$ \\
\hline Pent. & $\mathrm{AcOH}$ & 11.2297 & 13.4529 & $\mathrm{D}$ & $\mathrm{D}$ \\
\hline Pent. & $\mathrm{AcNH}_{2}$ & 7.0208 & 6.7911 & M & M \\
\hline Bz. & $\mathrm{AcOH}$ & 8.2846 & 8.2215 & $\mathrm{D}$ & $\mathrm{D}$ \\
\hline Pep. & Ethene & 5.9684 & 5.4634 & M & M \\
\hline Pyr. & Ethyne & 1.5647 & 1.5099 & $\mathrm{E}$ & $\mathrm{E}$ \\
\hline $\mathrm{MeNH}_{2}$ & Pyr. & 5.7620 & 1.8582 & M & $\mathrm{E}$ \\
\hline
\end{tabular}


눙 응

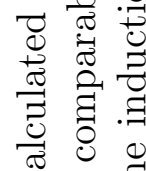

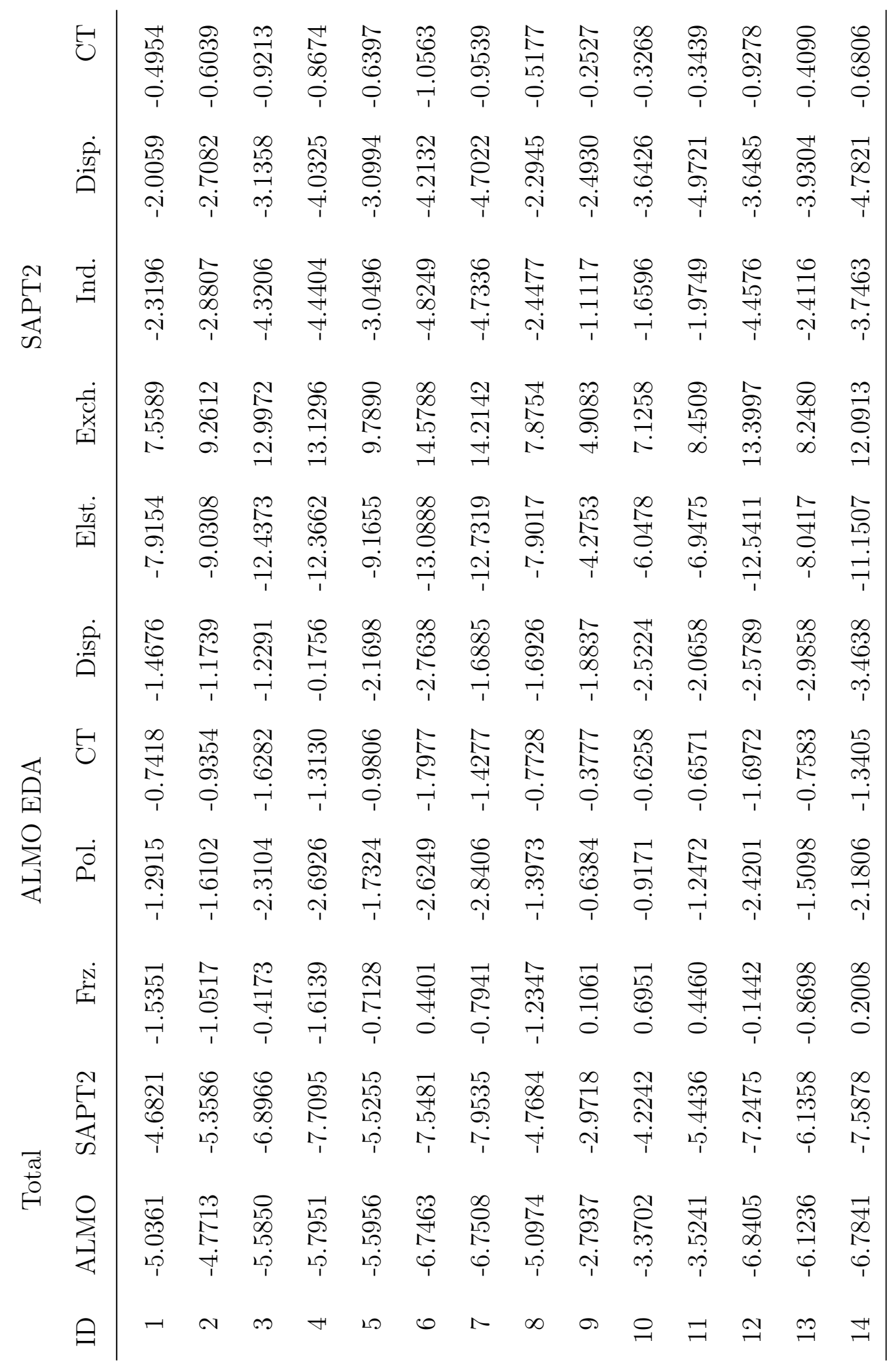
0.

要需包 0 弯 곻 马. .0 造 00 亦 द्व 궁 0 공 $\uplus$.

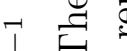
串里 त्ञ 덤 की दे

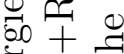
- 0 하 वै $\Xi$ : 。贯骂 잉 ॠ 记 ข 粪 क्य ०ै की

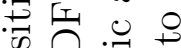
$\circ=$ 要专 80. O 600 园尔 $\forall 0$ 되를 응 $\ddot{N}$ है $:$ 事 입 


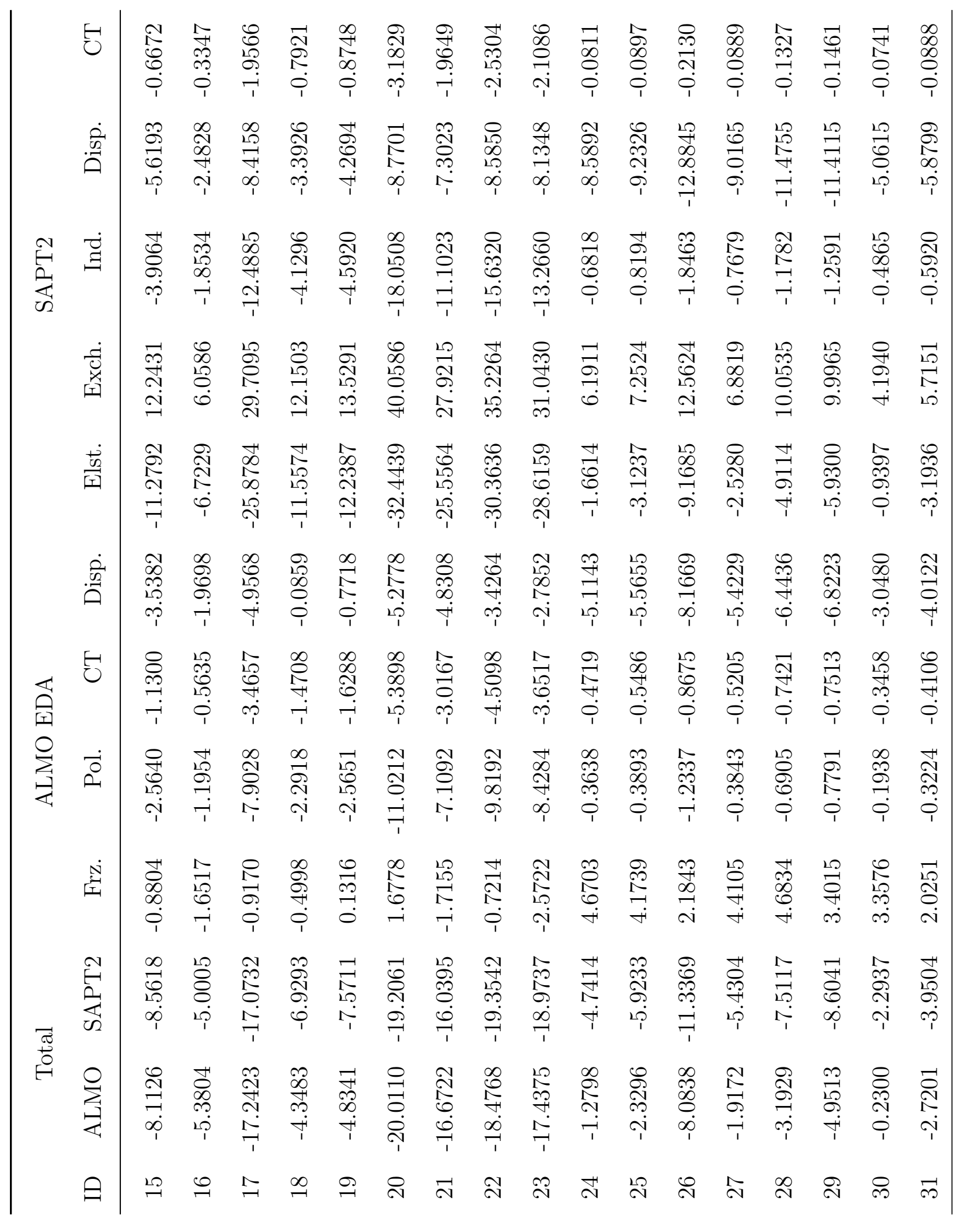




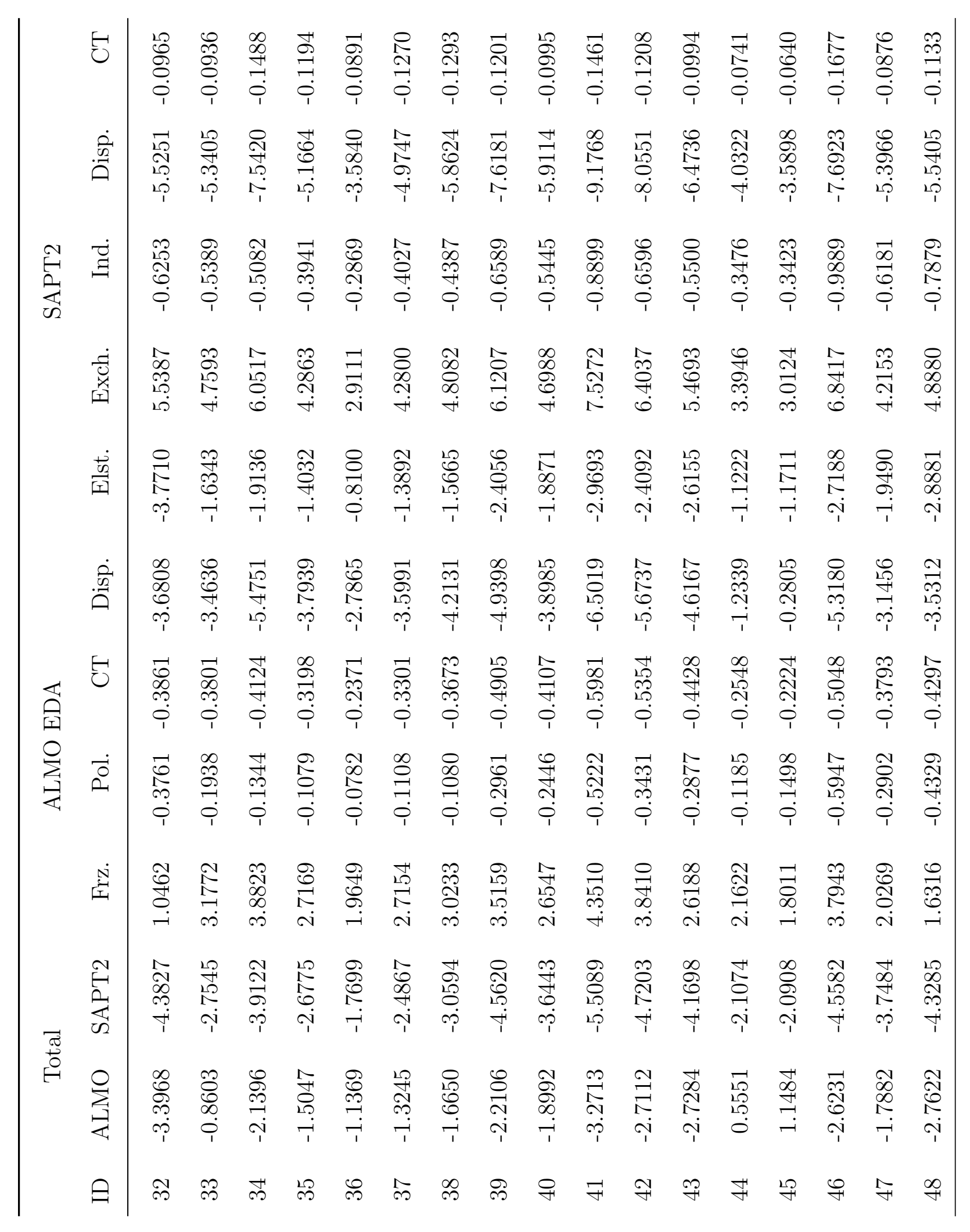




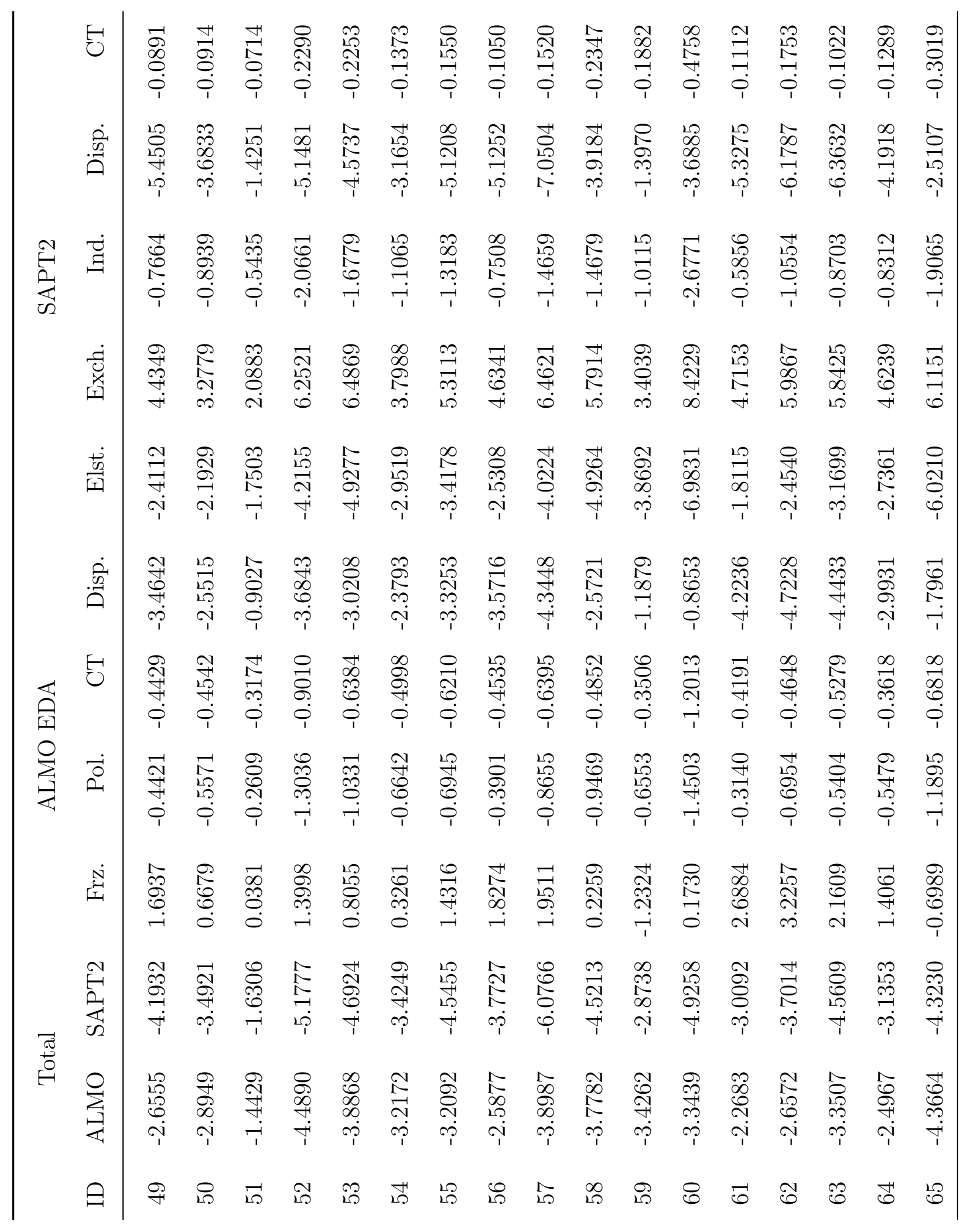




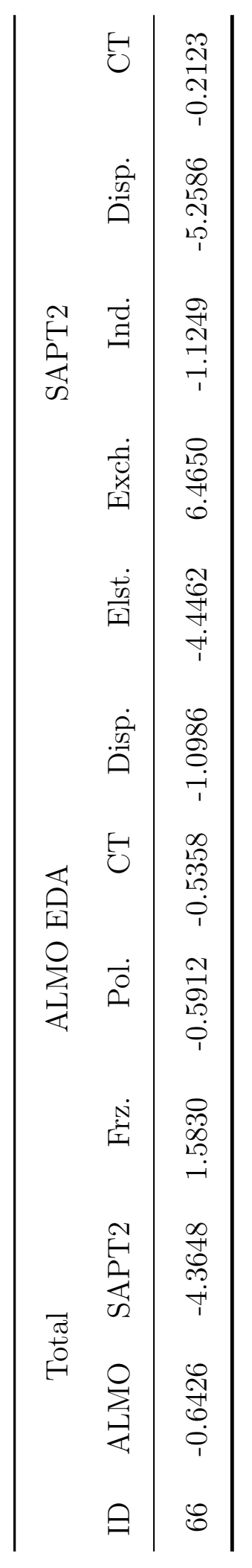




\section{Local thresholds, errors, and domain sizes}

Table S6: A list of default thresholds and cutoffs used in the blocked integrals, local exchange, and pairwise RPA portions of the program.

\begin{tabular}{lrl}
\hline Threshold & Value & Unit \\
\hline Integral precision, $\epsilon_{\text {Int. }}$ & $10^{-12}$ & - \\
Distance cutoff, AO domain & 15.0 & Bohr \\
MO domain, $\epsilon_{\mathrm{MO}}$ & $10^{-6}$ & - \\
Fitting domain, $\epsilon_{\text {Fit }}$ & 0.05 & - \\
Distance cutoff, pair RPA & 10.0 & Bohr \\
\hline
\end{tabular}
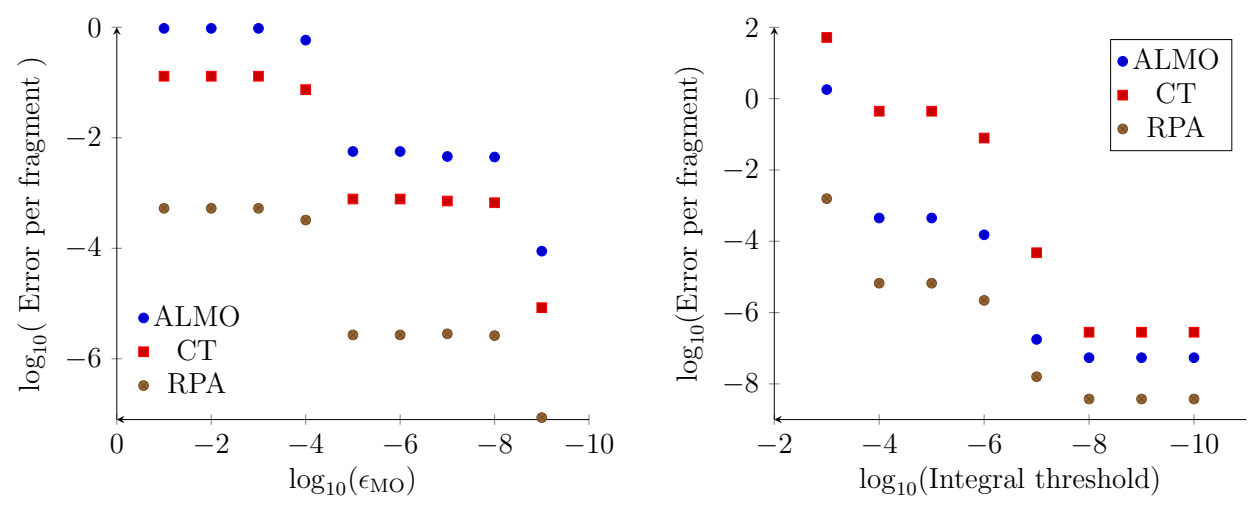

Figure S1: The error in the interaction as a function of the MO domain threshold (left) and integral screening threshold (right), taken as the average from calculations on a chain of 24 hydrogen fluorides and a cluster of ten water molecules. 
Table S7: Timings per iteration (in seconds) of the ALMO SCF routine with and without the local exchange approximation, calculated for various hydrogen fluoride chains using an aVDZ basis. The timings were averaged over all iterations (approximately 10), and all calculations were performed on a single CPU on the same node.

\begin{tabular}{rrr}
\hline No. of waters & Full & Local \\
\hline 3 & 0.08 & 0.03 \\
6 & 2.80 & 0.11 \\
9 & 18.90 & 0.18 \\
12 & 68.80 & 0.26 \\
15 & 260.40 & 0.35 \\
\hline
\end{tabular}

Table S8: Maximum and minimum orbital domain sizes (in units of number of fragments, including the fragment the orbital is on) for various clusters of $N$ water molecules, calculated using an aVDZ basis.

\begin{tabular}{rrrrrrr}
\hline \multicolumn{4}{c}{ Minimum } & \multicolumn{3}{c}{ Maximum } \\
$N$ & MO & AO & Fit & MO & AO & Fit \\
\hline 2 & 1 & 2 & 2 & 2 & 2 & 2 \\
3 & 1 & 3 & 3 & 3 & 3 & 3 \\
5 & 1 & 3 & 3 & 3 & 5 & 3 \\
10 & 1 & 4 & 4 & 5 & 10 & 4 \\
15 & 1 & 4 & 3 & 7 & 11 & 6 \\
20 & 1 & 5 & 5 & 9 & 20 & 7 \\
32 & 1 & 5 & 4 & 9 & 24 & 8 \\
\hline
\end{tabular}




\section{Geometries}

The coordinates for the S66 set CCSD/aVDZ geometries can be found in xyzfiles.tar.gz. The aVTZ calculations were performed using the equilibrium geometries from the original paper

[Rezac et al, JCTC, 2011]. Here we give images of the geometries of the systems for which energy curves were given in the main text (except He..Ne, as that would be fairly pointless). 

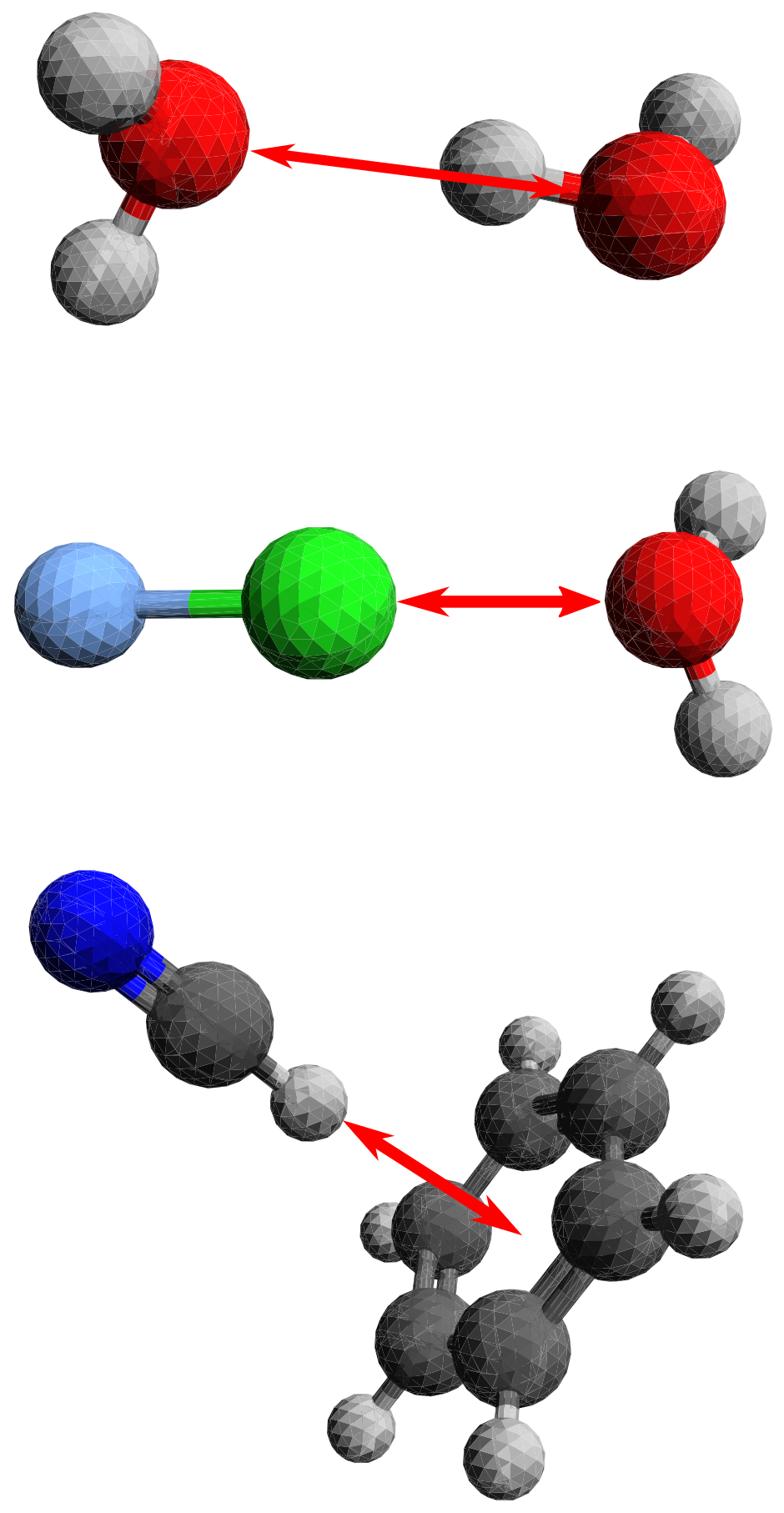

Figure S2: Equilibrium geometries, computed at the CCSD/aVDZ level, of the water dimer (top), $\mathrm{FCl} \cdots \mathrm{OH}_{2}$ (middle), and benzene with $\mathrm{HCN}$ (bottom). The scanned coordinate is also indicated in each case. 
Other files

datasets.zip (19.63 KiB)

view on ChemRxiv - download file 